\title{
三峡地区埃迪卡拉系至下寒武统地层中硒的含量 分布富集规律
}

\author{
田兴䂞 1,2 , 雒昆利 $2^{*}$ \\ 1. 中国科学院地理科学与资源研究所, 北京 100101 ; \\ 2. 中国科学院大学资源与环境学院, 北京 100049 \\ *通讯作者, E-mail: luokl@igsnrr.ac.cn
}

收稿日期: 2016-10-11; 接受日期: 2017-04-12; 网络版发表日期: 2017-05-25

国家自然科学基金项目(批准号: 41172310, 41472322)和国家重点基础研究发展计划项目(编号: 2014CB238906)资助

\begin{abstract}
摘要分析了三峡地区埃迪卡拉系至下寒武统 $\left(\mathrm{E}-\mathrm{C}_{1}\right)$ 地层中硒 $(\mathrm{Se})$ 的含量分布富集规律, 探讨了 $\mathrm{Se}$ 的显著和 非显著富集层的元素地球化学特征. 结果表明, $\mathrm{Se}$ 为 $\mathrm{E}-\mathrm{C}_{1}$ 地层中最富集(浓集)的元素,其富集系数 $\mathrm{EF}$ (与上地壳平 均元素含量相比)和浓集系数 $\mathrm{CC}$ (与同类岩性相比)均值分别为 26.97 和 48.04. 富集系数 $\mathrm{EF}^{\prime}(\mathrm{Al}$ 标准化之后的 $\mathrm{EF})$ 指示. Se为第二富集的元素 $\left(\mathrm{Se}_{\mathrm{EF}}=218.73\right)$, 低于 $\mathrm{Cd}\left(\mathrm{Cd}_{\mathrm{EF}}=288.46\right)$, 远高于第三富集的微量元素 $\mathrm{As}_{2}\left(\mathrm{As}_{\mathrm{EF}}=97.49\right)$. Se 在 $\mathrm{E}-\mathrm{C}_{1}$ 地层中含量范围为 $<10^{-5} \sim 30.08 \mathrm{ppm}$, 算数均值为 $1.35 \mathrm{ppm}$. 相对于南沱组 $\left(\mathrm{Se}_{\mathrm{EF}}=2.29\right), \mathrm{E}-\mathrm{C}_{1}$ 地层中 $\mathrm{Se}_{\mathrm{EF}}$ 值增 加11.78倍. 其 $\mathrm{EF}$ 均值呈现为水井沱组(92.58) > 岩家河组(54.45)>陡山沱组(24.72) > 灯影组(2.48)>石牌组(1.95) > 天 河板组下部(1.24). E- $\mathrm{C}_{1}$ 地层中 $\mathrm{Se}$ 的含量较服从自然对数正态分布, 表现出正偏态分布类型. Se显著富集(EF>10) 的层位主要包括陡II下部、陡II 上部、陡III顶部、陡IV、岩家河组底部和上部、水井沱组下部和上部. 元素地 球化学特征表明: Se显著富集层中 Se含量普遍受陆源碎屑的影响, 还受热液、火山碎屑、深部物源等单一或多 因素的共同作用. 此外, 黄铁矿对部分层位(陡II上部、陡IV和水井沱组上部)及有机质对部分层位(陡II下部和 水井沱组上部) 中 Se的富集具有促进作用. Se的非显著富集层(除陡 $\mathrm{I}$ 、水井沱组中部、石牌组和天河板组下部 外)中Se含量仍受陆源碎屑影响, 而其他的因素(热液、火山碎屑和深部物源等)的活动普遍较弱.
\end{abstract}

关键词埃迪卡拉系-下寒武统, 硒, 分布富集规律, 元素地球化学, 三峡地区

\section{1 引言}

扬子地台湖北三峡地区(图1)是中国南方埃迪卡拉 系标准剖面的所在地(赵自强等, 1985), 该地区埃迪卡 拉系至下寒武统( $\left.\mathrm{E}-\mathrm{C}_{1}, \mathrm{ca} .635 \sim 520 \mathrm{Ma}\right)$ 地层出露完整, 并且具有清晰的年代地层学(Condon等, 2005; Yin等,
2005; Liu等, 2009; Okada等, 2014)、岩石地层学(赵自 强等, 1985; 项礼文等, 1999)、生物地层学(赵自强等, 1985; McFadden等, 2009; 尹崇玉等, 2009; Liu等, 2013; Guo等, 2014)、同位素地层学(Jiang等, 2007; Ishikawa 等, 2008, 2013; Zhu等, 2013) 以及古环境(Ishikawa等, 2008; McFadden等, 2008; Scott等, 2008; 黄晶等, 2009)

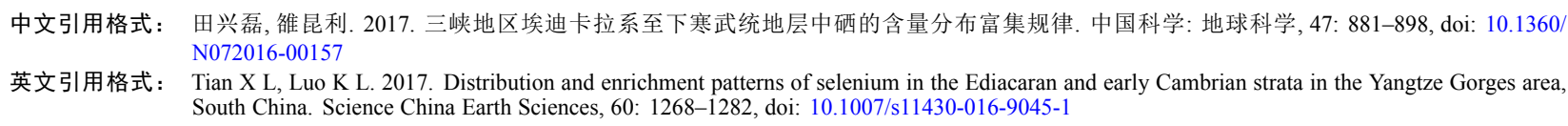

(C) 2017 《中国科学》杂志社

www.scichina.com 
和古地理(Jiang等, 2011)等研究背景. 其中, 对三峡地 区 $E-\epsilon_{1}$ 地层中微量元素的研究主要关注氧化还原敏 感微量元素(如Mo、U、V等)及稀土元素的地球化学 指示意义, 并运用其所表征的大气-海洋氧气的变化 来探究海洋氧化与生物演化之间的耦合关系(Scott等, 2008; Ling等, 2013), 或者关注氧化还原敏感元素及稀 土元素的含量变化来探究其沉积环境的变化(黄晶等, 2009; 田兴磊等, 2014; 闵斌等, 2014)及成矿元素如陡 山沱组顶部重晶石的成因及其地质指示意义(柳永清 等, 2009)等. 近期, Tian和Luo(2017)发现在三峡地区埃 迪卡拉系至下寒武统地层中微量元素硒( $\mathrm{Se})$ 、砷(As) 和钼(Mo)相对于成冰系南沱组显著富集, 其发生显著 富集的陡山沱组、岩家河组和水井沱组恰恰是该时 期生物发生大变革的层位, 并且, 该地区埃迪卡拉系 和下寒武统中 $\mathrm{Se} 、 \mathrm{As}$ 和Mo发生显著富集的层位与该 时期生物发生变革事件的层位吻合.

硒作为一类重要的生命必需微量元素, 是谷胱甘 肽过氧化物酶(GSH-Px)的重要组成(Rotruck等, 1973), 在预防心血管疾病、抗肿瘤、防癌和抗癌、抗氧化、 抗炎和抗感染等方面均有重要作用(Plant等, 2014). 此 外, $\mathrm{E}-\mathrm{C}_{1}$ 地层中的 $S e$ 对现生环境和人类健康具有重要 影响. 如Luo等(2004)报道陕南硒中毒区极高硒土壤中 硒的来源为当地埃迪卡拉纪和早寒武世高硒岩层的 风化. Ni等(2016)对湖南桃源县环境中的硒资源研究 发现, 当地富硒环境中硒的主要来源为早寒武世和埃 迪卡拉纪富硒的黑色岩系.

本文将系统分析三峡地区成冰系南沱组、埃迪 卡拉系及下寒武统沉积岩中微量元素硒的含量特征 及其分布富集规律, 并进一步揭示其显著和非显著富 集层的元素地球化学特征. 本研究可为探讨地球关键 生物演化时期的环境变化、探讨生物变革的环境因 素及揭示硒的富集规律提供科学数据.

\section{2 地质背景和样品}

三峡地区 $\mathrm{E}-\mathrm{C}_{1}$ 地层广泛分布于黄陵背斜周边地区 (图1a). 地层由老到新依次为埃迪卡拉系陡山沦组、 埃迪卡拉系灯影组、穿时地层(前寒武纪/寒武纪)岩 家河组、下寒武统水井沱组、石牌组、天河板组和 石龙洞组. 本文涉及的组具体如下.

陡山沱组总厚度约 $170 \mathrm{~m}$, 以盖帽白云岩与下伏成 冰系南沱组冰碛岩呈假整合接触(图1b). 陡山沱组自下 而上分为 4 个岩性段: I段(陡I)为一层全球性发育, 厚度 不等的碳酸盐岩地层, 称之为“帽碳酸盐岩”(Hoffman 等, 1998), 厚约 $2 \mathrm{~m}$, 岩性以灰色厚层状含砾微晶白云 岩为主; II段(陡II) 厚约 $100 \mathrm{~m}$, 深灰、黑色薄层状细晶 白云岩, 具水平微层理, 夹黑色页岩, 富含燧石结核; III段(陡III) 厚约 $50 \mathrm{~m}$, 白云岩、夹燧石层及燧石结核 和条带白云岩; IV段(陡IV)厚约 $13 \mathrm{~m}$, 黑色炭质页岩夹 泥晶白云岩透镜体 ( $(\mathrm{Lu}$ 等, 2012).

灯影组位于陡山沱组之上, 与下伏陡山沱组整合 接触, 厚约 400m. 岩性三分明显(赵自强等, 1985; Chen 等, 2013), 下部为灰色、灰白色内碎屑白云岩, 含核 形石及燧石条带和结核, 称蛤蟆井段, 厚约 $30 \mathrm{~m}$; 中部 为黑色薄板状微晶石灰岩, 含石膏层, 称石板滩段, 厚 约 $270 \mathrm{~m}$; 上部主要为灰白色块状白云岩, 微晶或粗晶 白云岩, 内碎屑白云岩, 夹燧石层, 含燧石团块和燧石 结核, 产同生角砾岩, 称白马沱段, 厚约 $100 \mathrm{~m}$ (Chen等, 2013).

岩家河组厚约 $40 \mathrm{~m}$, 以深灰色中薄层状白云岩夹 薄层硅质岩出现为底, 与灯影组白马沱段整合接触. 其主要岩性特征自下而上为: I段厚约 $15 \mathrm{~m}$, 黑色中薄 层硅质岩夹砂质白云岩及灰黑色页岩; II段厚约 $2 \mathrm{~m}$, 为灰白色中层状含硅磷质砾屑白云岩; III段厚约 $18 \mathrm{~m}$, 深灰色中薄层微晶灰岩夹黑色粉砂质页岩; IV段厚约 $4 \mathrm{~m}$, 黑色页中层状灰岩; $\mathrm{V}$ 段厚约 $2 \mathrm{~m}$, 为灰白色中层状 含硅磷质砾屑灰岩 (Guo等, 2014). 水井沱组厚约 $40 \mathrm{~m}$, 以黑色页岩和页岩为主, 夹石灰岩透镜体及中厚层石 灰岩(项礼文等, 1999). 石牌组厚约 $100 \mathrm{~m}$, 以黄绿色薄 层粉砂岩和页岩为主. 天河板组厚约 80 至 $90 \mathrm{~m}$, 以灰白 色泥晶灰岩为主(项礼文等, 1999).

三峡地区埃迪卡拉系至下寒武统地层具有 精确的年代地层学约束, 陡山沱组的年代界定于 635 551Ma(Condon等, 2005), 水井沱组底部U-Pb年龄 为(526.4 \pm 5.4$) \mathrm{Ma}$ (Okada等, 2014)(图1b).

本次研究涉及采自 7 个典型剖面的岩石样品 397 件, 地层厚度约900m(图1b). 其中, 在王丰岗剖面采集 南沱组底部样品,在田家园子剖面采集南沱组和陡山 沱组样品, 在九龙湾剖面采集陡II上部样品, 在黄牛岩 剖面采集陡III上部和陡IV样品,在雾河剖面采集灯影 组样品,在滚子坳剖面采集岩家河组和水井沱组样品, 在夷陵剖面采集水井沱组、石牌组和天河板组样品. 

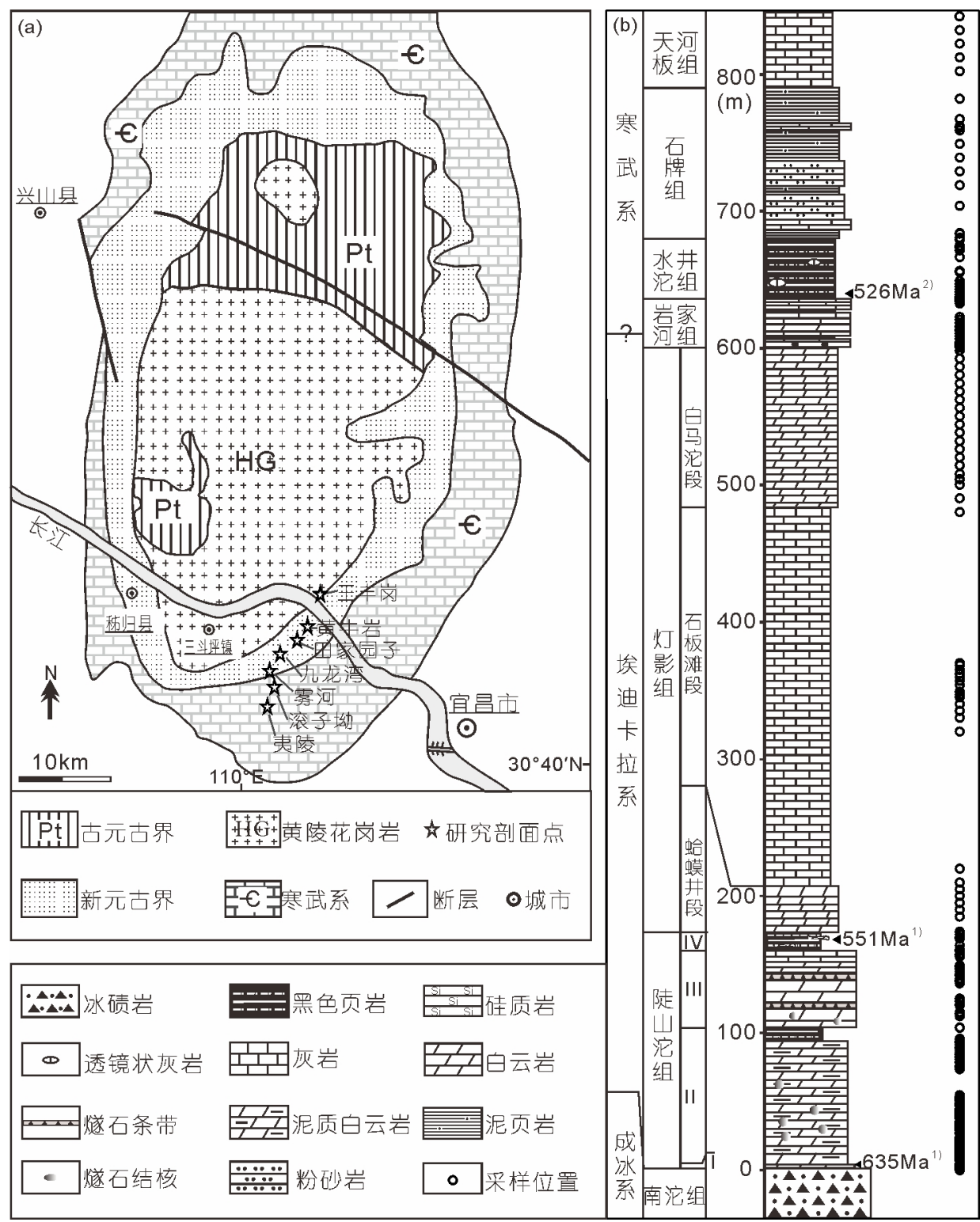

图 1 研究剖面位置示意图及综合地层柱状图

(a) 三峡地区地质简图; (b) 综合地层柱状图. 地质年代数据来源: 1) Condon等(2005); 2) Okada等(2014)

具体的综合岩性柱状图和样品位置见图 $1 \mathrm{~b}$ 和网络版 附录1(http://earthcn.scichina.com, 下同). 为尽量确保采 集的样品能反映埃迪卡拉系至下寒武统地层的整体 特征, 我们在岩性变化较大的组段如陡山沱组、岩家 河组进行密集采样, 采样间隔约为 $0.5 \mathrm{~m}$, 而在岩性相 对单一的组段如灯影组以及在因风化或覆盖而不能 在约 $0.5 \mathrm{~m}$ 的距离上采集样品水井沱组、石牌组及天 河板组的部分层位进行稀疏采样, 采样间距5.0 20m.

\section{3 实验方法}

\section{1 样品前处理}

野外采样过程中尽量选择比较新鲜的岩石剖面 或岩石面, 避开风化、含后期白云岩或石英脉、有后 期溶蚀及重结晶现象的岩石. 室内处理时, 去掉岩石 样品表面杂质(泥土、植物、苔藓等), 用去离子水冲 洗, 自然风干. 随后对冰碛岩及沉积岩采样不同的前 
处理. 对冰碛岩, 轻微敲碎后过 10 目篮, 尽量挑选不含 颗粒的冰碛岩基质, 粉碎至 200 目, 装在塑料自封袋中 待分析; 而对沉积岩, 尽量挑选新鲜的样品粉碎至 200 目,装在塑料自封袋中待分析.

\section{2 样品分析}

常量元素和微量元素(包括稀土元素)的分析测 定: 岩石样品 (200目)用三酸 $\left(\mathrm{HNO}_{3}-\mathrm{HF}-\mathrm{HClO}_{4}\right)$ 消解 法消解(Luo, 2011; 田兴磊等, 2014). 元素含量使用 Perkin Elmer公司生产的电感耦合等离子体光谱仪 (ICP-OES)及电感耦合等离子体质谱仪(ICP-MS)测定. $\mathrm{Na} 、 \mathrm{Al} 、 \mathrm{~K} 、 \mathrm{Ti} 、 \mathrm{Mn} 、 \mathrm{Fe} 、 \mathrm{Li} 、 \mathrm{Ni} 、 \mathrm{Sr} 、 \mathrm{~V}$ 和 $\mathrm{Zn}$ 的 检测限为 $0.1 \mathrm{ppb}, \mathrm{Mg} 、 \mathrm{P}$ 和 $\mathrm{Ca}$ 的检测限为 $1.0 \mathrm{ppb}, \mathrm{Cd}$ 、 Co、 Tl、Ga、In、Rb、Th、U、Bi、Mo和Be的检出 限为 $0.0001 \mathrm{ppb}$. 砷和硒的含量使用原子荧光光度计法 测定, 具体消解方法见(Niu和Luo, 2011; Ni等, 2016). 硒 含量的检测限为 $0.01 \mathrm{ppb}$, 砷含量的检测限为 $0.05 \mathrm{ppb}$. 总有机碳(TOC)按照国标中沉积岩中总有机碳的测定 方法 $(\mathrm{GB} / \mathrm{T} 19145,2003)$ 测定. 分析误差优于 $10 \%$. 以上 测试均在中国科学院地理科学与资源研究所理化分 析中心测定.

\section{3 质量控制}

准确的实验数据是研究的重要基础, 质量保证则 是实验的最基本要求. 本研究从野外岩石样品的采集 到实验室内实验流程和样品的分析测试均严格遵循野 外和室内操作规范. 实验中所使用的玻璃器血均提前 在 $20 \%$ 左右的硝酸中过夜浸泡, 使用前用去离子水洗 净烘干. 实验中使用的聚四氟乙烯(PTEF)烧杯和烧杯 盖, 在使用前需用自来水和去离子水清洗干净, 并置于 $20 \%$ 的硝酸溶液中煮沸 $20 \mathrm{~min}$, 之后用去离子水冲洗干 净后烘干备用. 为控制分析数据的质量, 所有的分析过 程中均以国家标准物质: 土壤(GBW07401, GBW07403, GBW07406) ${ }^{1)}$ 和岩石(GBW07107, GBW07112) 检验分 析结果的准确性, 以平行样、空白实验以及重复测量 等手段检测分析结果的平行性和稳定性.

\section{4 结果和讨论}

\section{1 硒的含量分布富集规律}

主量元素、微量元素及稀土元素的含量及相 关指标列于网络版附录1 6中. 本研究采用富集系 数 $\mathrm{EF}$ (Enrichment Factor)、富集系数 $\mathrm{EF}^{\prime}$ 和浓集系数 $\mathrm{CC}$ (Concentration Coefficient)探讨岩石样品中元素的 富集程度. 按照富集情况将微量元素的富集情况分 为6类: 显著富集 $(\mathrm{EF}>10)$ 、强富集 $(10>\mathrm{EF}>5)$ 、弱富集 $(5>E F>2)$ 、正常 $(2>E F>0.5)$ 以及亏损 $(0.5>E F)$. 对 $C C$ 和 $\mathrm{EF}^{\prime}$ 也按照此分类标准.

富集系数 $\mathrm{EF}$ 为样品中元素 $X$ 的含量相对于上地壳 或平均页岩(PAAS)中元素 $X$ 的丰度值, 本文中统一选 用上地壳 $(\mathrm{UCC})$ 的元素含量做为对比标准, 公式 $X_{\mathrm{EF}}=$ $X_{\text {样品 }} X_{\mathrm{UCC}}$, 其中 $\mathrm{UCC}$ 中元素的平均含量引自(Taylor和 McLennan, 1985; McLennan, 2001).

因为沉积岩物质成分变化大, 根据高于或低于地 壳(或页岩)值认定微量元素的富集亏损情况会产生偏 差, 比如一些生物成因的碳酸盐岩或蛋白石等矿物会 起到稀释作用(Tribovillard等, 2006). 为了消除这种影 响将元素 $X$ 通过 $\mathrm{Al}$ 或 $\mathrm{Th}$ 标准化来计算富集系数 $\mathrm{EF}^{\prime}$, 计 算公式为 $X_{\mathrm{EF}}=(X / \mathrm{Al})_{\text {样品 }} /(X / \mathrm{Al})_{\mathrm{UCC}}$.

浓集系数 $(\mathrm{CC})$ 为样品中某一元素 $X$ 的含量与此类 样品的同类岩性中的此元素 $X$ 含量的比值, 计算公式

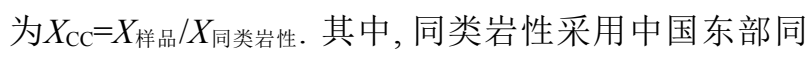
类岩性. 其含量引自迟清华和鄢明才(2007).

结果显示, 相对于上地壳微量元素均值, $\mathrm{Se}$ 是 三峡地区埃迪卡拉系至下寒武统中所研究的 23 种微 量元素中最富集的元素, 其富集系数 $\left(\mathrm{Se}_{\mathrm{EF}}=26.97\right)$ (网 络版附录4)显著强于其他微量元素, 是第二富集元 素 $\mathrm{Cd}\left(\mathrm{Cd}_{\mathrm{EF}}=10.66\right)$ 的 2.53 倍, 并且 $\mathrm{Se}$ 在埃迪卡拉纪至 早寒武世地层中富集系数 $\mathrm{EF}$ 的变化较大, 其 $\mathrm{EF}$ 的差 值, 即最大富集系数 $\mathrm{EF}_{\text {max }}$ 与最小富集系数 $\mathrm{EF}_{\min }$ 的差 值为 601.2 (网络版附录4). 浓集系数均值表明 $\mathrm{Se}$ 在三 峡地区埃迪卡拉系至下寒武统中具有最高的 $\mathrm{CC}$ 值 $\left(\mathrm{Se}_{\mathrm{CC}}=48.04\right)$ (表 1 , 网络版附录5), 是第二浓集微量元素 $\mathrm{Cd}\left(\mathrm{Cd}_{\mathrm{CC}}=10.70\right)$ 的 4.49 倍. 富集系数 $\mathrm{EF}^{\prime}$ 指示, $\mathrm{Se}$ 为第 二富集的元素 $\left(\mathrm{Se}_{\mathrm{EF}}=218.73\right.$, 网络版附录6), 稍弱于

\footnotetext{
1) 中国地质科学院地球物理地球化学勘查研究所. 2003. 标准物质证书-土壤成分分析标准物质. 中国, 廊坊

2) 中国地质科学院地球物理地球化学勘查研究所. 2003. 标准物质证书-岩石成分分析标准物质. 中国, 廊坊
} 
表 1 三峡地区埃迪卡拉系至下寒武统不同组段中 $\mathrm{Se}$ 的富集程度及 $\mathrm{Eu} / \mathrm{Eu}^{*}$ 和 $\mathrm{Ce} / \mathrm{Ce}{ }^{*}$ 的值 ${ }^{\mathrm{a}}$

\begin{tabular}{|c|c|c|c|c|c|c|c|}
\hline \multicolumn{3}{|c|}{ 地层系统 } & $\mathrm{Se}_{\mathrm{EF}}$ & $\mathrm{Se}_{\mathrm{CC}}$ & $\mathrm{Se}_{\mathrm{EF}^{\prime}}$ & $\mathrm{Eu} / \mathrm{Eu}^{*}$ & $\mathrm{Ce} / \mathrm{Ce}^{*}$ \\
\hline \multicolumn{3}{|c|}{ 天河板组下部 } & 1.24 & 0.84 & 51.08 & 0.87 & 0.65 \\
\hline \multicolumn{3}{|c|}{ 石牌组 } & 1.95 & 1.04 & 10.31 & 1.00 & 0.87 \\
\hline \multirow[t]{5}{*}{ 下寒武统 } & \multicolumn{2}{|l|}{ 水井沱组 } & 92.58 & 4.76 & 124.71 & 1.12 & 0.86 \\
\hline & \multicolumn{2}{|l|}{ 岩家河组 } & 54.45 & 12.17 & 265.50 & 1.16 & 0.57 \\
\hline & & 白马沱段 & 2.57 & 2.96 & 1013.14 & 1.03 & 0.26 \\
\hline & 灯影组 & 石板滩段 & 2.04 & 1.45 & 394.20 & 1.09 & 0.26 \\
\hline & & 蛤蟆井段 & 3.43 & 4.29 & 175.46 & 1.07 & 0.55 \\
\hline \multirow[t]{6}{*}{ 埃迪卡拉系 } & \multicolumn{2}{|l|}{ 灯影组均值 } & 2.48 & 2.56 & 665.95 & 1.06 & 0.30 \\
\hline & \multirow{4}{*}{ 陡山沱组 } & 陡IV & 249.70 & 11.56 & 328.56 & 1.38 & 0.90 \\
\hline & & 陡 III & 8.82 & 6.74 & 473.89 & 1.12 & 0.62 \\
\hline & & 陡 II & 13.14 & 88.66 & 81.42 & 1.22 & 0.73 \\
\hline & & 陡I & 1.36 & 4.19 & 14.25 & 1.42 & 0.92 \\
\hline & \multicolumn{2}{|l|}{ 陡山沱组均值 } & 24.72 & 68.97 & 148.02 & 1.22 & 0.73 \\
\hline \multicolumn{3}{|c|}{ 埃迪卡拉系-下寒武统均值 } & 26.97 & 48.04 & 218.73 & 1.18 & 0.67 \\
\hline 成冰系 & \multicolumn{2}{|l|}{ 南沱组 } & 2.29 & 0.19 & 2.84 & 1.21 & 0.82 \\
\hline
\end{tabular}

a) $\mathrm{Eu} / \mathrm{Eu}^{*}=2 \mathrm{Eu}_{\mathrm{N}} /\left(\mathrm{Sm}_{\mathrm{N}}+\mathrm{Gd}_{\mathrm{N}}\right) ; \mathrm{Ce} / \mathrm{Ce}^{*}=2 \mathrm{Ce}_{\mathrm{N}} /\left(\mathrm{La}_{\mathrm{N}}+\mathrm{Pr}_{\mathrm{N}}\right)$, 公式参考Bau和Dulski(1996), N代表后太古代澳大利亚平均页岩(PAAS)标准化, PAAS 的数据引自 Nance和Taylor(1976)

$\mathrm{Cd}\left(\mathrm{Cd}_{\mathrm{EF}}=288.46\right)$, 远高于第三富集的微量元素 $\operatorname{As}\left(\operatorname{As}_{\mathrm{EF}}=97.49\right)$.

Se在埃迪卡拉系至下寒武统中含量变化范围为 $<10^{-5} \sim 30.08 \mathrm{ppm}$, 算数均值为 $1.35 \mathrm{ppm}$. Se含量在陡IV 黑色页岩中较之前层位中的 $\mathrm{Se}$ 的含量和富集程度均 显著增加(表1和2), 最高值出现在岩家河组底部(网络 版附录2). Se在三峡地区埃迪卡拉系至下寒武统组间 的平均富集系数 $\mathrm{EF}$ 呈现为水井沱组 $>$ 岩家河组 $>$ 陡山 沱组 $>$ 灯影组 $>$ 石牌组 $>$ 天河板组下部(表1). 其中在水 井沱组、岩家河组和陡山沱组中的 $\mathrm{EF}$ 均值远高于其 他组. 在整个涉及的E- $\mathrm{C}_{1}$ 地层中, Se的EF均值为 26.97 , 是下伏成冰系南沱组 $\left(\mathrm{Se}_{\mathrm{EF}}=2.29\right)$ 的11.78倍.

在埃迪卡拉系和下寒武统中 $\mathrm{Se}$ 的富集系数 $\mathrm{EF}$ 的 具有强烈的波动变化, 其中发生显著富集的层位(表2) 包括: 陡II下部, 即盖帽白云岩之上2.4 10.8m的层位; 陡II上部, 即陡 II 上部距陡II/陡III界线约 $30 \mathrm{~m}$ 的层位; 陡III顶部距陡III/陡IV 界线约 $0.2 \mathrm{~m}$ 的层位; 陡IV 整段; 岩家河组底部 $0.9 \mathrm{~m}$ 的层位; 岩家河组上部 $24.05 \mathrm{~m}$ 的层 位; 水井沱组下部 $11.0 \mathrm{~m}$ 的层位; 水井沱组上部 $9 \mathrm{~m}$ 的层 位. 此外, $\mathrm{CC}$ 和 $\mathrm{EF}^{\prime}$ 在这些显著富集层位中同样出现峰
值(图2; 表 1 , 网络版附录 5 和 6 ).

\section{2 硒的含量分布模式}

直方图是最常用的描述数据分布的图解之一, 并 被广泛的应用的地学领域(Zhang等, 2005; Rawlins等, 2012). 它由并排的直方条组成, 可以直观的显示数据 的单峰或多峰分布模式. 直方图两侧偏离主体的部分 数据为尾翼数据, 在直方图的一端或两端可直接检测 出离群数据即偏离数据主体的数据点.

频率分布直方图表明, 三峡地区埃迪卡拉系-下寒 武统微量元素Se的含量数据属非正态分布, 而是表现 出正偏态分布类型(图3a), 表明在低浓度范围内的值 占大多数 $(<2.5 \mathrm{ppm})$, 而偏离主体的尾翼数据主要在右 侧, 即高浓度的数据为尾翼数据, 与上文提及的硒的显 著富集层位主要集中在个别层位如埃迪卡拉系陡山沱 组IV段、岩家河组下部和水井沱组下部和上部一致.

那么, 该套地层中Se的含量服从哪种类型的分布 呢? 下面我们运用分位数图(简称Q-Q图)检验其分布 模式. 分位数是指某一期望分布函数的反函数值. Q-Q 图是 $x$ 轴为样本的分位数, $y$ 轴为某一期望分布形式的 


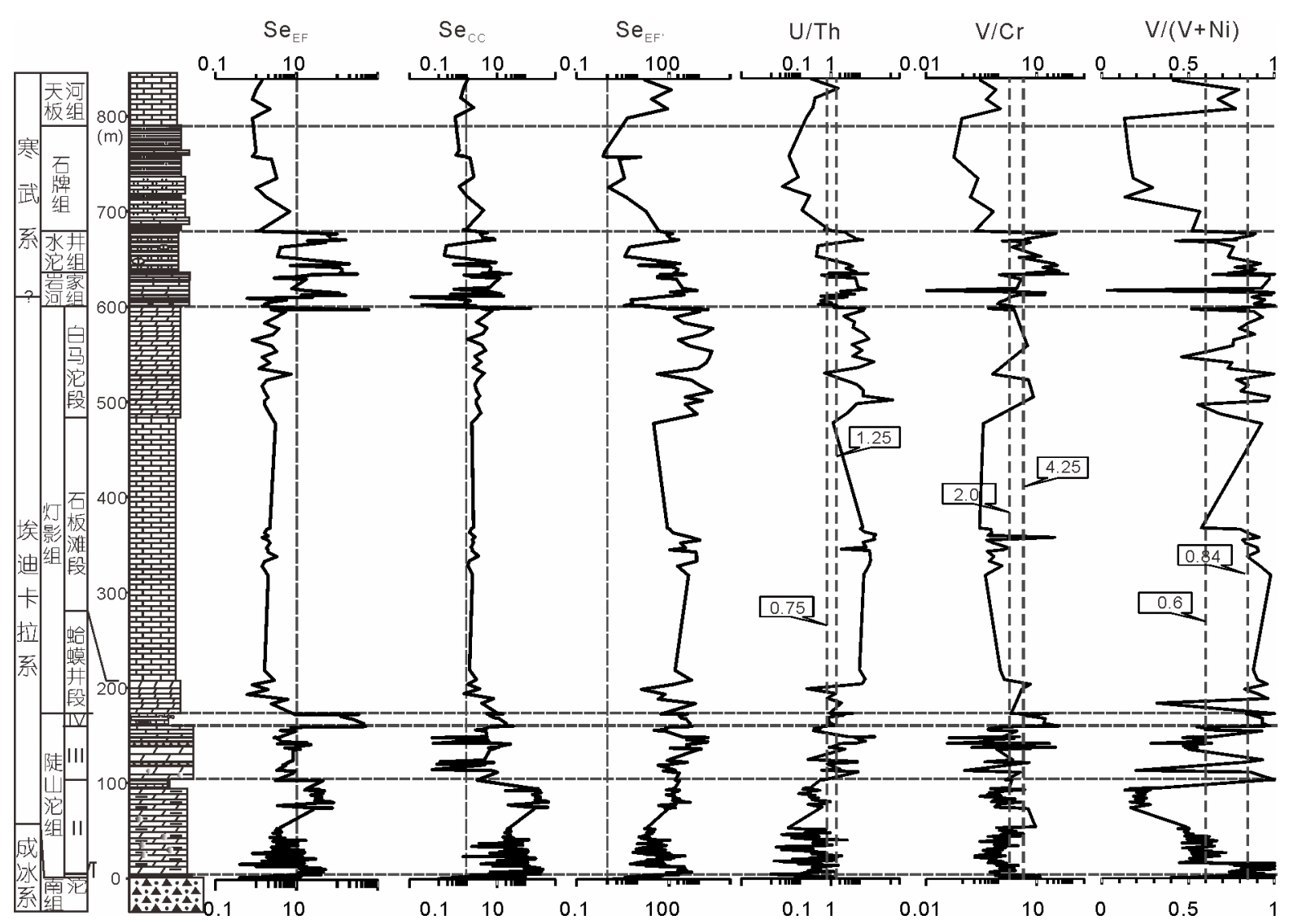

图 2 三峡地区南沱组顶部至天河板组下部地层中Se的富集程度及相应的古环境指标的变化曲线

表 2 三峡地区埃迪卡拉系至下寒武统Se显著富集 $(\mathrm{EF}>10)$ 的层位及相应的参数值

\begin{tabular}{|c|c|c|c|c|c|c|c|c|}
\hline & $\begin{array}{c}\text { 水井沱组 } \\
\text { 上部 }\end{array}$ & $\begin{array}{c}\text { 水井沱组 } \\
\text { 下部 }\end{array}$ & $\begin{array}{c}\text { 岩家河组 } \\
\text { 上部 }\end{array}$ & $\begin{array}{c}\text { 岩家河组 } \\
\text { 底部 }\end{array}$ & 陡IV全部 & 陡III顶部 & 陡II上部 & 陡II下部 \\
\hline $\mathrm{Se}(\mathrm{ppm})$ & $0.92 \sim 8.08$ & $0.35 \sim 15.82$ & $0.14 \sim 8.05$ & $0.66 \sim 30.08$ & $4.80 \sim 25.08$ & $0.97 \sim 2.55$ & $0.17 \sim 4.0$ & $0.55 \sim 2.53$ \\
\hline $\mathrm{Se}_{\text {均值 }}(\mathrm{ppm})$ & 3.83 & 7.2 & 2.12 & 6.69 & 12.49 & 1.76 & 1.76 & 1.39 \\
\hline $\mathrm{Se}_{\mathrm{EF}}$ & 76.63 & 144.09 & 42.3 & 133.73 & 249.7 & 35.2 & 35.27 & 27.79 \\
\hline $\mathrm{Se}_{\mathrm{CC}}$ & 4.8 & 6.67 & 7.9 & 33.26 & 11.56 & 25.14 & 230.32 & 191.89 \\
\hline $\mathrm{Se}_{\mathrm{EF}}$ & 126.87 & 174.11 & 199.38 & 663.36 & 328.56 & 279.75 & 162.38 & 269.64 \\
\hline $\mathrm{Eu} / \mathrm{Eu}^{*}$ & $1.03 \sim 1.14$ & $0.96 \sim 1.96$ & $0.68 \sim 2.72$ & $1.01 \sim 1.56$ & $1.06 \sim 1.89$ & $1.40 \sim 2.21$ & $0.97 \sim 1.33$ & $1.17 \sim 4.04$ \\
\hline $\mathrm{Eu} / \mathrm{Eu}^{*}$ 均值 & 1.09 & 1.18 & 1.17 & 1.17 & 1.38 & 1.81 & 1.12 & 1.59 \\
\hline $\mathrm{Ce} / \mathrm{Ce}^{*}$ & $0.70 \sim 0.92$ & $0.77 \sim 0.90$ & $0.39 \sim 0.72$ & $0.36 \sim 0.60$ & $0.83 \sim 0.99$ & 0.73 & $0.48 \sim 0.82$ & $0.67 \sim 0.91$ \\
\hline $\mathrm{Ce} / \mathrm{Ce}^{*}$ 均值 & 0.84 & 0.83 & 0.57 & 0.50 & 0.90 & 0.73 & 0.71 & 0.78 \\
\hline $\mathrm{U} / \mathrm{Th}$ & $3.3 \sim 11.55$ & $2.33 \sim 7.19$ & $0.46 \sim 17.01$ & $1.24 \sim 13.22$ & $0.8 \sim 5.22$ & $0.80 \sim 1.20$ & $0.09 \sim 0.97$ & $0.10 \sim 1.29$ \\
\hline $\mathrm{U} / \mathrm{Th}$ 均值 & 5.82 & 4.54 & 4.48 & 5.33 & 2.27 & 1.0 & 0.32 & 0.43 \\
\hline $\mathrm{V} / \mathrm{Cr}$ & $1.19 \sim 33.73$ & $5.5 \sim 71.01$ & $0.01 \sim 42.72$ & $0.5 \sim 2$ & $3.53 \sim 40.93$ & $5.61 \sim 5.91$ & $0.29 \sim 5.98$ & $0.55 \sim 2.70$ \\
\hline $\mathrm{V} / \mathrm{Cr}_{\text {均值 }}$ & 12.39 & 32.66 & 8.20 & 1.18 & 12.96 & 5.76 & 1.57 & 1.43 \\
\hline $\mathrm{V} /(\mathrm{V}+\mathrm{Ni})$ & $0.43 \sim 0.89$ & $0.73 \sim 0.95$ & $0.03 \sim 1$ & $0.52 \sim 0.81$ & $0.83 \sim 0.98$ & $0.92 \sim 0.94$ & $0.13 \sim 0.95$ & $0.74 \sim 1.0$ \\
\hline $\mathrm{V} /(\mathrm{V}+\mathrm{Ni})$ 均值 & 0.73 & 0.86 & 0.87 & 0.68 & 0.92 & 0.93 & 0.25 & 0.87 \\
\hline
\end{tabular}



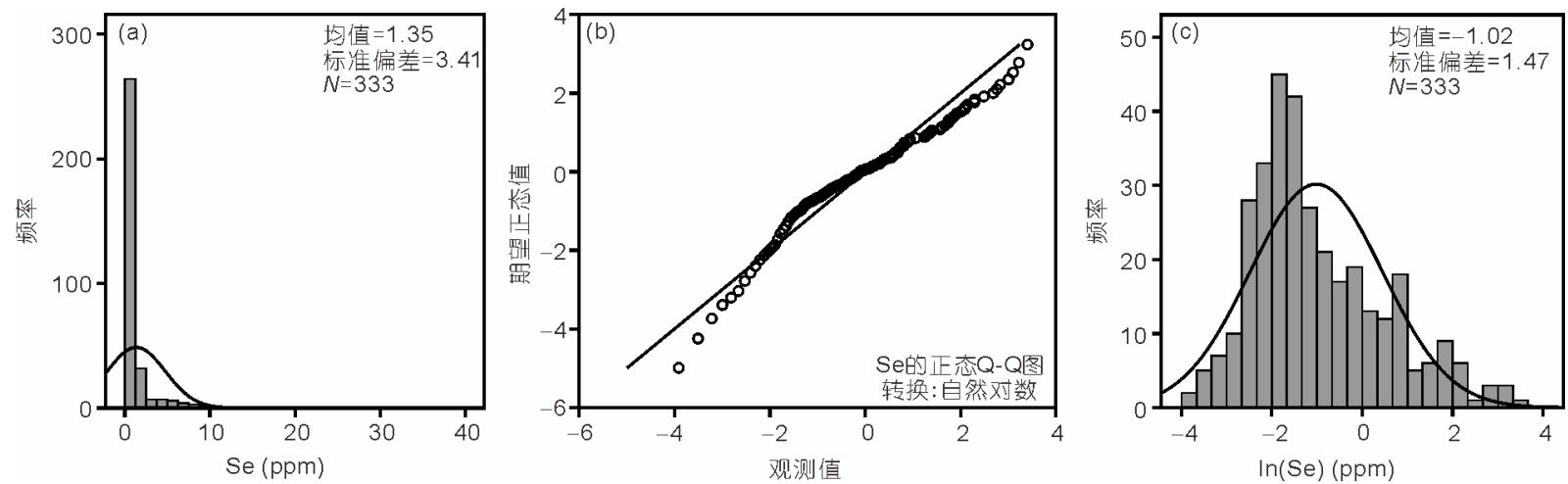

图 3 三峡地区埃迪卡拉系-下寒武统地层中 Se含量的分布模式检验

(a) 频率分布直方图; (b) Q-Q图检验; (c) 自然对数值的频率分布直方图

分位数. 若数据服从某一分布模式如正态分布、对数 分布、自然对数分布或其他分布模式, 则投点应该是 呈一条围绕第一象限对角线的直线. Q-Q图可以直观 检验元素是否服从正态或对数正态分布, 在地学领域 应用广泛(Zhang等, 2005; Rawlins等, 2012). 本文中, 对 三峡地区埃迪卡拉系至下寒武统微量元素 $\mathrm{Se}$ 的含量 数据进行检验, 发现 $\mathrm{Se}$ 较符合自然对数正态分布模式 (图3b). Se含量的自然对数值的频率分布直方图同样 显示其较符合正态分布的类型(图3c).

\section{3 硒显著富集层和非显著富集层的元素地球化 学特征}

\subsection{1 陡II下部硒显著富集层}

大多数沉积岩中, 总有部分微量元素来自于陆源 碎屑 (Tribovillard等, 2006). 在陆源细碎屑沉积岩中, $\mathrm{Al} 、 \mathrm{Th}$ 和 $\mathrm{Ti}$ 等元素主要来自陆源风化物, 且在次生作 用中不易迁移, 因此非常适合用于进行陆源碎屑检验 (Tribovillard等, 2006). 如果某个元素的含量与Al、Th 或Ti的含量表现出良好的正相关关系, 说明该元素的 含量主要受陆源碎屑控制.

陡II整段陆源碎屑指示元素 $\mathrm{Al}$ 与 $\mathrm{Th}$ 和 $\mathrm{Ti}$ 的相关 性分析(图4a和b)显示, 陡II整段中 $\mathrm{Al}$ 与 Th和 Ti线性关 系较好, 具有显著正相关关系 $\left(R_{\mathrm{Al}-\mathrm{Th}}=0.73, R_{\mathrm{Al}-\mathrm{Ti}}=0.92\right.$, $P<0.01, n=169)$, 表明陡II中陆源碎屑物质的源区稳定. 在陡II整段中, $\mathrm{Se}$ 与 $\mathrm{Al}$ 和 $\mathrm{Th}$ 均表现出显著正相关关系 $\left(R_{\mathrm{Se}-\mathrm{Al}}=0.19, R_{\mathrm{Se}-\mathrm{Th}}=0.33, P<0.05, n=169\right)$, 指示在该段中 $\mathrm{Se}$ 的含量受陆源碎屑的影响显著.

相比海水提供的硒而言, 热液能提供大量的硒
(Auclair等, 1987), 在热液环境下形成的硅酸盐矿物和 硫化物集合体中的硒含量可高达1640ppm(Rouxel等, 2004). 热液最显著的特征是具有非常明显的Eu的正异 常(Bau和Dulski, 1996). Eu是稀土元素中唯一可以从三 价态 $\mathrm{Eu}^{3+}$ 还原为二价态 $\mathrm{Eu}^{2+}$ 的元素(Brookins, 1989), Eu 的正异常往往与高温 $\left(>250^{\circ} \mathrm{C}\right)$ (Sverjensky, 1984)和还 原性热液流体相关(Michard和Albarède, 1986; Olivarez 和Owen, 1991; Bau和Dulski, 1996). 在陡II下部Se显著 富集层中, 具有轻微的 $E u$ 的正异常 $\left(\mathrm{Eu} / \mathrm{Eu}^{*}\right.$ 平均=1.59)(表 2), 其中在盖帽白云岩之上 $6.5 \mathrm{~m}$ 处具有明显的 $\mathrm{Eu}$ 的正 异常 $\left(\mathrm{Eu} / \mathrm{Eu}^{*}=4.03\right.$ ) (网络版附录3). 全球范围内海底热 液流体普遍具有LREE富集和具有异常高Eu的正异常 的特点(丁振举等, 2000). 在该 $\mathrm{Eu}$ 明显正异常的层位, 稀土配分表现出HREE略富集的特点(田兴否等, 2014), 与海底热液流的特点不尽一致. 海底热液流体进入海 洋环境之后会被海水稀释 1000 至 10000 倍, 甚至更高 (Nozaki, 1997). 此外, 受热液喷出口附近的金属离子 吸附作用的影响, 稀土配分呈现出热液和海水混合的 模式(van Kranendonk等, 2003), 热液的LREE富集特征 被海水严重稀释, 甚至受金属离子的沉淀作用导致轻 重稀土重新分馏(Bau和Dulski, 1996), 而呈现出HREE 富集, 接近海水的Y/Ho值(Alibo和Nozaki, 1999), Eu正 异常得以保留, La正异常, 低 $\sum$ REE的特征. 而微弱的 正异常指示沉积物受热液改造作用较小, 一种可能性 是热液与海水的混合, 造成当时的海水中具有 $\mathrm{Eu}$ 的正 异常, 并且这种正异常被自生沉积物保存下来.

此外, 在此段富集层位中, $\mathrm{P}$ 含量亦显著增加(网络 版附录1). P的最高值(13690.0ppm)出现在 $\mathrm{Eu}$ 具有显著 
正异常的层位中. 海洋中P的主要有陆源和上升流两 种来源(Filippelli, 2008). 陡II中 $\mathrm{P}$ 与 $\mathrm{Al}$ 表现出微弱的负 相关关系 $(R=-0.14)$ (图 $5 \mathrm{a})$, 指示该段中 $\mathrm{P}$ 含量不受陆源 碎屑的影响. 因此, 该段 $\mathrm{P}$ 的来源主要为深部海水的上 涌. 由于该富集层位中 $\mathrm{P}$ 与 $\mathrm{Se}$ 均无显著正相关关系(图 $5 b)$, 因此 $S e$ 不具有深海来源.

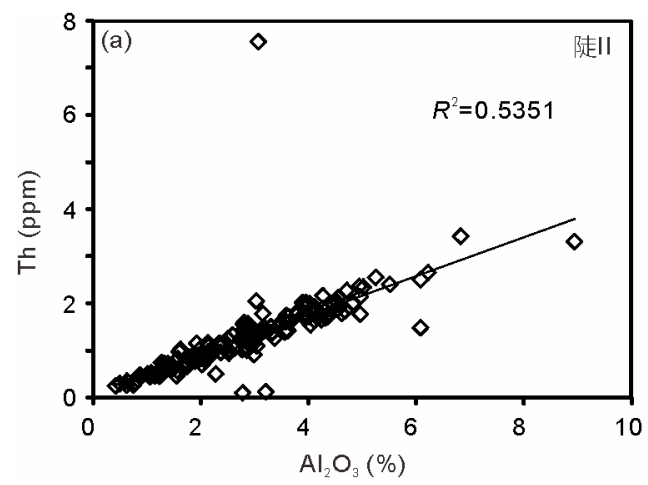

硒的产出往往与火山作用相关(Floor和 RománRoss, 2012). 研究表明, 高的 $\mathrm{Rb} / \mathrm{K}$ 的值是古代高度风 化物的特征 (Plank 和 Langmuir, 1998), 而低的 Rb/K 的 值则是富火山物质沉积物的典型特征或者是沉积物 经过钾的交代(如沸石成岩作用导致的结果)(Plank和 Langmuir, 1998). 将陡II下部Se显著富集层样品落入在

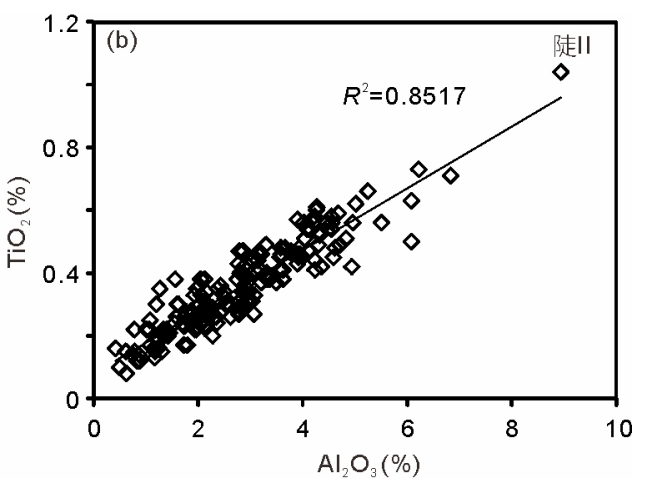

图 4 陡II中 $\mathrm{Al}$ 与 $\mathrm{Th}(\mathrm{a})$ 和 Ti(b) 的相关性分析
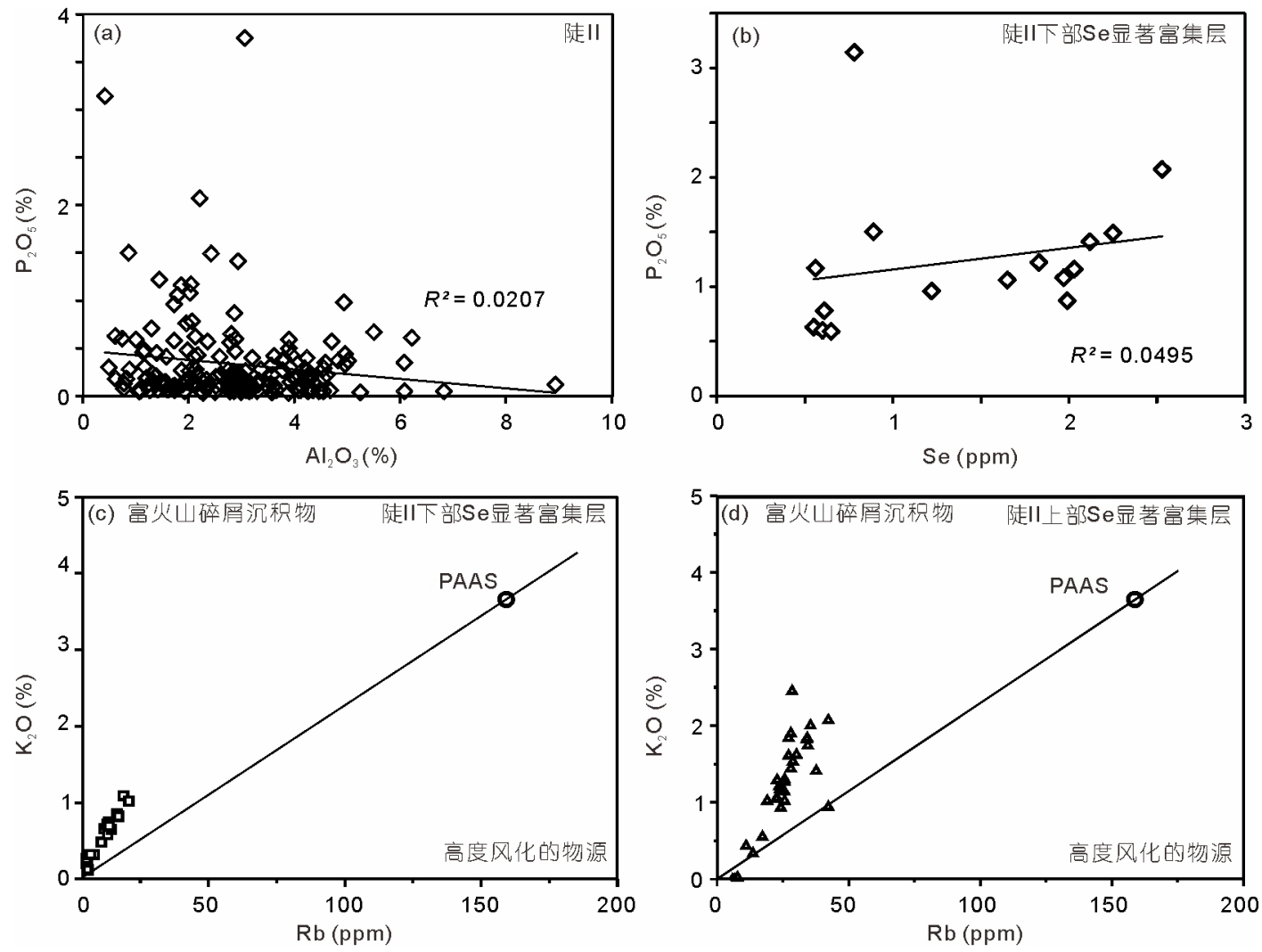

图 5 陡II中陆源碎屑及火山碎屑的判别图解

(a) 陡II中 P 与 $\mathrm{Al}$ 的相关性分析; (b) 陡II下部Se显著富集层中 $\mathrm{P}$ 与 Se的相关性分析; (c) 陡II下部Se显著富集层中 $\mathrm{K}_{2} \mathrm{O}-\mathrm{Rb}$ 判别图解; (d) 陡II上部 $\mathrm{Se}$ 显著富集层中 $\mathrm{K}_{2} \mathrm{O}-\mathrm{Rb}$ 判别图解 
$\mathrm{K}_{2} \mathrm{O}-\mathrm{Rb}$ 图解中 (图 $5 \mathrm{c}$ ), 样品具有较低的 $\mathrm{Rb} / \mathrm{K}$ 的值, 落在 富火山碎屑沉积物一侧, 推测 $\mathrm{Se}$ 可能具有火山碎屑源. 此外, 陡II中 $\mathrm{Se}$ 与 $\mathrm{TOC}$ 表现出显著正相关 $\left(R_{\mathrm{Se}-\mathrm{TOC}}=0.66\right.$, $P<0.01, n=26$ ), 表明该段中有机质含量的升高促进 $\mathrm{Se}$ 的富集.

\subsection{2 陡II上部硒显著富集层}

此段 $\mathrm{Se}$ 含量受陆源碎屑控制(讨论见4.3.1). Eu异 常表现出轻微的正异常, 未见明显的正异常, 表明热 液作用不明显. 另外, 陡II中P含量的来源主要为深部 海水(讨论见4.3.1). 而在陡II上部中 $\mathrm{Se}$ 与 $\mathrm{P}$ 仅具有轻微 的正相关性 $(R=0.09)$, 因此排除 $\mathrm{Se}$ 的深部来源. 该富集 层中 $\mathrm{Se}$ 与 TOC 具有较强的正相关 $\left(R_{\mathrm{TOC}-\mathrm{Se}}=0.63\right)$, 表明有 机质对 $\mathrm{Se}$ 的富集具有一定的促进作用. 同时, 该富集 层中 $\mathrm{S}$ 与 $\mathrm{Fe}$ 具有显著正相关 $\left(R_{\mathrm{Fe}-\mathrm{S}}=0.68, P<0.01, n=30\right)$, 证明 $\mathrm{S}$ 主要是黄铁矿硫. 黄铁矿是 $\mathrm{Se}$ 的重要载体( Large 等, 2014), 而 $\mathrm{Se}$ 与 $\mathrm{S}$ 仅具有轻微的正相关 $(R=0.15)$, 表明 黄铁矿的存在对该层位中 $\mathrm{Se}$ 的显著富集有一定的贡 献, 但不显著. 此外, $\mathrm{K}_{2} \mathrm{O}-\mathrm{Rb}$ 图解(图 $5 \mathrm{~d}$ )指示陡 $\mathrm{II}$ 上部 $\mathrm{Se}$ 显著富集层中受火山碎屑的影响.

\subsection{3 陡III顶部硒显著富集层}

陡 III整段中, $\mathrm{Al}$ 与 $\mathrm{Th}$ 具有显著的正相关关系 $\left(R_{\mathrm{Al}-\mathrm{Th}}=0.88, P<0.01, n=32\right)$, 表明陡III中陆源碎屑物 质的源区稳定. 而陡III与陡 II中 $\mathrm{Al} / \mathrm{Th}(\% / \mathrm{ppm})$ 的均值 分别为 0.7 和 1.36 , 表明陡III和陡II陆源碎屑物质的成 分发生了变化, 陡III的陆源碎屑来自于一个相对富 Th的源区.

陡 III 中 $\mathrm{Se}$ 与 $\mathrm{Al}$ 表现出显著正相关 $\left(R_{\mathrm{Se}-\mathrm{Al}}=0.42\right.$, $P<0.05, n=32)$, 指示 $\mathrm{Se}$ 含量受陆源碎屑输入的作用显 著. 该段中 $\mathrm{P}$ 与 $\mathrm{A} 1$ 和 $\mathrm{Th}$ 均不具有正相关性 $\left(R_{\mathrm{P}-\mathrm{Al}}=-0.16\right.$; $\left.R_{\mathrm{P}-\mathrm{Th}}=0.12\right)$, 表明 $\mathrm{P}$ 不具有陆源碎屑的来源, 而是受深 部物源的控制. 而 $\mathrm{Se}$ 和 $\mathrm{P}$ 同样没有表现出明显正相关 $\left(R_{\mathrm{P}-\mathrm{Se}}=0.09\right)$, 因此 $\mathrm{Se}$ 不受深部物源的影响.

在 Se显著富集的陡山沱组III段顶部样品DST3-0 中, $\mathrm{Eu}$ 具有较明显的正异常 $\left(\mathrm{Eu} / \mathrm{Eu}^{*}=2.21\right)$ (网络版附录 3 ), 暗示热液作用的存在. 而DST3-0的稀土配分曲线呈 现出无明显的轻重稀土富集的特征 $\left((\mathrm{La} / \mathrm{Yb})_{\mathrm{N}}=1.02\right)$ (网 络版附录3), 均与典型的海底热液流的LREE富集及明 显的 $\mathrm{Eu}$ 的正异常的特点有所差别. 与陡II下部的探讨 一致, 说明热液流并非直接影响沉积物, 而是经过与海
水的混合后, Eu正异常得以保留, La正异常, 低 $\sum$ REE 的特征. 热液活动也会显著影响沉积物或沉积岩中微 量元素的含量. 通常海底热液携带相当高含量的 $\mathrm{Ba}$ 、

$\mathrm{Sr} 、 \mathrm{~Pb}$ 和Zn等金属元素(Marchig等, 1982), 造成这些 元素在热液活动区域形成的沉积物或沉积岩中含量 明显增加. 在陡山沱组III段顶部 $\mathrm{Se}$ 显著富集层中Ba、

$\mathrm{Sr} 、 \mathrm{~Pb}$ 和 $\mathrm{Zn}$ 具有明显增加的现象(网络版附录 2 ). 此 外, 具有较高的 $R b / K$ 的值, 指示未受明显的火山物质 的影响.

\subsection{4 陡IV硒显著富集层}

陡 IV 黑色页岩中 $\mathrm{Ce} / \mathrm{Ce}^{*}$ 的变化区间为 $0.83 \sim 0.99$, 均值为 0.90 (表 2$),(\mathrm{La} / \mathrm{Ce})_{\mathrm{N}}$ 的范围为 $1.06 \sim 1.38$, 均值为 1.21 , 而页岩中 $(\mathrm{La} / \mathrm{Ce})_{\mathrm{N}}$ 在 $0.5 \sim 1.5, \mathrm{Ce} / \mathrm{Ce}^{*}$ 在 $0.84 \pm 0.12$ 时 指示其构造环境为大陆边缘(Murray等, 1990; Murray, 1994), 因此, 陡IV黑色页岩沉积构造背景为大陆边缘, 这与前人研究一致(Jiang等, 2011). 而陡IV整段中 $\mathrm{Se}$ 与 $\mathrm{Al}$ 表现出一定的正相关关系 $\left(R_{\mathrm{Se}-\mathrm{Al}}=0.48\right)$ (图6a), 表明 $\mathrm{Se}$ 含量在一定程度上受到陆源碎屑的影响, 但不显著.

陡IV中 $\mathrm{Fe}$ 与 $\mathrm{S}$ 具有显著正相关 $\left(R_{\mathrm{Fe}-\mathrm{S}}=0.92, P<0.01\right.$, $n=12)$, 证明该段黑色页岩中 $\mathrm{S}$ 主要是黄铁矿硫. 该段 中 $\mathrm{Se}$ 与 $\mathrm{S}$ 表现出一定的正相关关系 $\left(R_{\mathrm{Se}-\mathrm{S}}=0.40\right)$, 但不显 著, 指示黄铁矿对陡IV的Se的富集有一定的贡献, 但 不是 $\mathrm{Se}$ 富集的主要因素.

$\mathrm{P}$ 与 $\mathrm{Al}$ 和 $\mathrm{Th}$ 不具有显著正相关关系 $\left(R_{\mathrm{P}-\mathrm{Al}}=0.39\right.$; $\left.R_{\mathrm{P}-\mathrm{Th}}=0.45\right)$, 推测其受陆源输入的影响较弱, 主要来源 为上升流(Filippelli, 2008). 陡IV沉积阶段恰好是陡山 沱期第三次海侵期(McFadden等, 2008). 其中 $\mathrm{Se}$ 与 $\mathrm{P}$ 具 有较强的负相关 $\left(R_{\mathrm{Se}-\mathrm{P}}=0.47\right)$, 表明 $\mathrm{Se}$ 不具有深部来源.

$\mathrm{K}_{2} \mathrm{O}-\mathrm{Rb}$ 图解指示陡山沱组IV段黑色页岩落在富 火山碎屑沉积物区(图6b), Se具有一定的火山碎屑源. 陡IV 黑色页岩中 $\mathrm{Se}$ 与 TOC表现出较强的负相关关系 $\left(R_{\mathrm{Se}-\mathrm{TOC}}=0.64\right)$, 表明三峡地区陡 IV 的黑色页岩中高的 有机质含量并未对 $\mathrm{Se}$ 的显著富集有所贡献.

陡IV黑色页岩中普遍具有 Eu的轻微的正异常(表 2). 需要注意的是, 在极度缺氧硫化 (euxinic)的环境 中如现代黑海中Eu也能达到稳定的二价态(Sverjensky, 1984). 氧化还原敏感元素 $U, V$ 和 Mo 的是氧化 还原环境的很好的指示元素, 它们与 TOC仅在缺氧 环境下形成的沉积物或者沉积岩中具有较好的正相 关关系(Tribovillard等, 2006). 陡IV 黑色页岩中U、 
Mo、 V与 TOC 的相关性依次为 $0.12 、 0.35$ 和 -0.66 , 指 示陡IV 黑色页岩的沉积环境为硫化的环境. 沉积物 中 $\mathrm{U} / \mathrm{Al} 、 \mathrm{Mo} / \mathrm{Al}$ 及 $\mathrm{V} / \mathrm{Al}$ 的值通过与标准页岩(PAAS和 黑海静海沉积物) 的比较, 在现生沉积物和古代页岩 中判别沉积环境时应用广泛 (Algeo和Maynard, 2004; $\mathrm{Pi}$ 等, 2013). 此外, 陡 $\mathrm{IV}$ 黑色页岩中 $\mathrm{Al}$ 与 $\mathrm{Ti}$ 具有显著 正相关 $\left(R_{\mathrm{Al}-\mathrm{Ti}}=0.98, P<0.01, n=12\right)$, 表明 $\mathrm{Al}$ 具有正常的 碎屑源来源. 因此, 运用 $\mathrm{Al}$ 标准化探讨沉积环境是合 适的. 陡 $\mathrm{IV}$ 黑色页岩中 $\mathrm{U} / \mathrm{Al} 、 \mathrm{~V} / \mathrm{Al}$ 及 $\mathrm{Mo} / \mathrm{Al}$ 的变化范 围分别为1.75 8.06、41.18 194.25和0.37 24.88ppm/\%, 均值分别为 $4.01 、 101.01$ 和 $12.52 \mathrm{ppm} / \%$, 均高于黑海 静海沉积物的值 $(\mathrm{U} / \mathrm{Al}=3.3 \mathrm{ppm} / \%$; $\mathrm{V} / \mathrm{Al}=28.8 \mathrm{ppm} / \%$; $\mathrm{Mo} / \mathrm{Al}=4.3 \mathrm{ppm} / \%$ ), 同样表明陡IV 黑色页岩的沉积环 境为极为缺氧的硫化的环境. 此外, 陡IV 黑色页岩中 热液特征元素 $\mathrm{Ba} 、 \mathrm{~Pb}$ 和 $\mathrm{Zn}$ 的富集系数 $\mathrm{EF}^{\prime}$ 的值分别为 $4.61 、 6.88$ 和 $3.0, \mathrm{~Pb}$ 表现出强富集, 而 $\mathrm{Ba}$ 和 $\mathrm{Zn}$ 为弱富 集. 因此, 不能排除硫化环境对Eu正异常的干扰. 故推
测陡IV 黑色页岩中 Se受热液作用不明显.

\subsection{5 岩家河组硒显著富集层}

硅质岩中 $\mathrm{Ce} / \mathrm{Ce}{ }^{*}$ 和 $(\mathrm{La} / \mathrm{Ce})_{\mathrm{N}}$ 比值可以区分构造环 境(Murray等, 1990; Murray, 1994). 其中, $\mathrm{Ce} / \mathrm{Ce}^{*}$ 的值为 $0.60 \pm 0.11$ 和 $(\mathrm{La} / \mathrm{Ce})_{\mathrm{N}}$ 比值范围在1.0 2.5, 指示构造环境 为盆地底部. 岩家河组中硅质岩中 $\mathrm{Ce} / \mathrm{Ce}{ }^{*}$ 和 $(\mathrm{La} / \mathrm{Ce})_{\mathrm{N}}$ 比 值(表3)表明岩家河组沉积时期其构造环境为盆地底 部. 岩家河组 $\mathrm{Se}$ 与 $\mathrm{Al}$ 未显示出显著正相关 $\left(R_{\mathrm{Se}-\mathrm{Al}}=0.28\right)$, 表明陆源碎屑对该组Se的富集有一定的贡献,但不是 $\mathrm{Se}$ 富集的主要因素, 同时也说明岩家河组Se的富集还 受其他因素的影响.

硅质岩中的常量元素 $\mathrm{Al} 、 \mathrm{Fe} 、 \mathrm{Mn}$ 等可以用来区 分硅质岩的成因类型和构造沉积环境. Boström(1983) 根据现代热水沉积物和古代类似的沉积物中的常量 元素特点, 提出热水成因的硅质岩明显富 $\mathrm{Si} 、 \mathrm{Fe} 、 \mathrm{Mn}$, 而贫 $\mathrm{Al}$ 和 $\mathrm{Ti}$, 可以用 $\mathrm{Al} /(\mathrm{Al}+\mathrm{Fe}+\mathrm{Mn})$ 及 $\mathrm{Fe} / \mathrm{Ti}$ 的比值来区
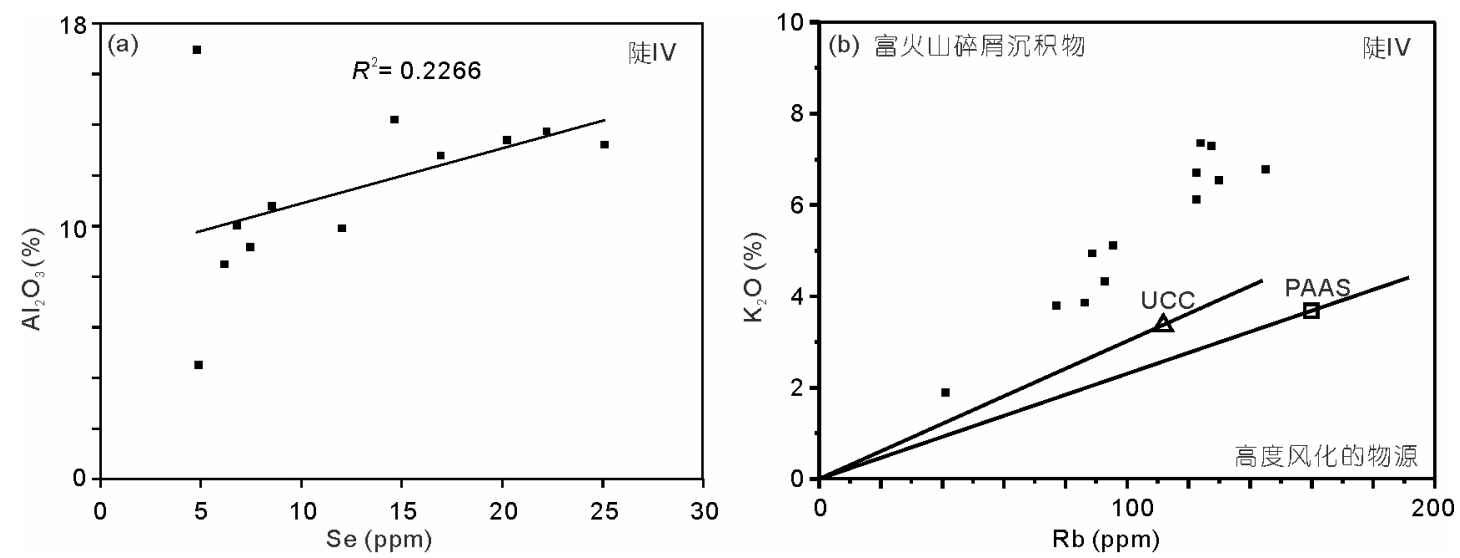

图 6 陡IV中陆源碎屑及火山碎屑的判别图

(a) $\mathrm{Se}$ 与 $\mathrm{Al}$ 的相关性分析; (b) $\mathrm{K}_{2} \mathrm{O}-\mathrm{Rb}$ 判别图解

表 3 岩家河组硅质岩中 Se的富集程度及相应的参数值

\begin{tabular}{cccccccccccc}
\hline 样品号 & 地层 & $\mathrm{Mn}(\%)$ & $\mathrm{Al}(\%)$ & $\mathrm{Fe}(\%)$ & $\mathrm{Fe} / \mathrm{Ti}$ & $\begin{array}{c}\mathrm{Al} /(\mathrm{Al}+ \\
\mathrm{Fe}+\mathrm{Mn})\end{array}$ & $\mathrm{Se}_{\mathrm{EF}}$ & $\mathrm{Se}_{\mathrm{CC}}$ & $\mathrm{Se}_{\mathrm{EF}}$ & $(\mathrm{La} / \mathrm{Ce})_{\mathrm{N}}$ & $\mathrm{Ce} / \mathrm{Ce}{ }^{*}$ \\
\hline YJH-8 & 岩家河III段 & 0.02 & 0.17 & 0.60 & 33.44 & 0.22 & 17.8 & 0.34 & 819.16 & 2.14 & 0.51 \\
YJH-14 & 岩家河I段 & 0.03 & 0.34 & 0.57 & 23.92 & 0.36 & 13.2 & 0.25 & 313.23 & 1.63 & 0.70 \\
YJH-16 & 岩家河I段 & 0.02 & 0.19 & 0.35 & 19.44 & 0.33 & 0.60 & 0.01 & 26.03 & 1.44 & 0.66 \\
YJH-21 & 岩家河I段 & 0.04 & 0.49 & 0.65 & 15.50 & 0.42 & 1.40 & 0.03 & 22.86 & 1.35 & 0.71 \\
YJH-23 & 岩家河I段 & 0.03 & 0.78 & 0.71 & 13.09 & 0.51 & 4.20 & 0.08 & 43.39 & 1.76 & 0.59 \\
YJH-25-1 & 岩家河I段 & 0.05 & 0.79 & 0.98 & 20.42 & 0.43 & 109.0 & 2.10 & 1110.97 & 1.68 & 0.60 \\
\hline
\end{tabular}


分热水沉积物和一般海水沉积物, 认为当比值分别小 于 0.35 和大于 20 时属于热水成因. 岩家河组I段底部 三件硅质岩(YJH-25-1、YJH-23和YJH-21)中 Fe/Ti的比 值均大于 0.35 (表 3), 除 $\mathrm{YJH}-25-1$ 的 $\mathrm{Al} /(\mathrm{Al}+\mathrm{Fe}+\mathrm{Mn}$ ) 的值 略高于 20 之外, 其他两个硅质岩中 $\mathrm{Al} /(\mathrm{Al}+\mathrm{Fe}+\mathrm{Mn})$ 的值 均低于 20 (表3), 表明岩家河组I段底部硅质岩非热水 成因. 硅质岩 $\mathrm{YJH}-16$ (岩家河组下部)中 $\mathrm{Al} /(\mathrm{Al}+\mathrm{Fe}+\mathrm{Mn}$ ) 略小于 0.35 , 而 $\mathrm{Fe} / \mathrm{Ti}$ 略小于 20 . 岩家河组上部(YHJ-14) 中 $\mathrm{Al} /(\mathrm{Al}+\mathrm{Fe}+\mathrm{Mn})$ 的值略高于 0.35 , 而 $\mathrm{Fe} / \mathrm{Ti}$ 大于 20 . 岩 家河组上部 $(\mathrm{YJH}-8)$ 中 $\mathrm{Al} /(\mathrm{Al}+\mathrm{Fe}+\mathrm{Mn})$ 的值小于 0.35 , 而 $\mathrm{Fe} / \mathrm{Ti}$ 值大于 20 , 为典型的热水硅质岩.

岩家河组硅质岩成因上从下至上发生了明显的 变化. 进一步将硅质岩落在 Al-Fe-Mn判别图解上(图 7), 指示岩家河组底部YJH-25-1和YJH-21 为非热水和 非生物沉积硅质岩, 而YJH-23 为典型的生物硅质岩. 岩家河组上部(YJH-16、YJH-14和YJH-8)为热水硅质 岩. 岩家河组底部Se显著富集层中 Se不具有热液来源, 而从岩家河组I段下部开始沉积受到了热液影响(图7), 推测岩家河组上部 $\mathrm{Se}$ 显著富集层中 $\mathrm{Se}$ 具有热液来源.
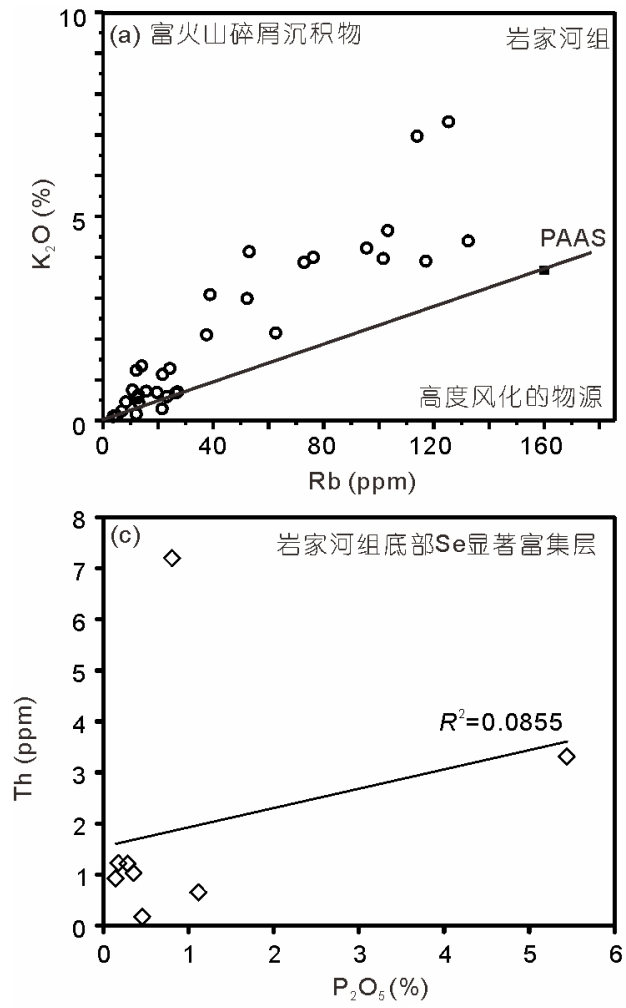

图 8 岩家河组陆源碎屑及火山碎屑的判别图解

(a) $\mathrm{K}_{2} \mathrm{O}-\mathrm{Rb}$ 判别图解; (b) (d) P与相关元素的相关性分析 
影响.

$\mathrm{K}_{2} \mathrm{O}-\mathrm{Rb}$ 图解(图8a)指示岩家河组上部仍有部分火 山碎屑物质的影响. 岩家河组上部Se显著富集层中, $\mathrm{P}$ 与 $\mathrm{Al}$ 和 Th无明显正相关关系 $\left(R_{\mathrm{P}-\mathrm{Al}}=0.04 ; R_{\mathrm{P}-\mathrm{Th}}=0.06\right)$, 并且 $\mathrm{Se}$ 与 $\mathrm{P}$ 无显著正相关关系 $(R=0.13)$, 推测 $\mathrm{Se}$ 不受深 部物源的影响.

\subsection{6 水井沱组硒显著富集层}

根据水井沱组黑色页岩中 $\mathrm{Ce} / \mathrm{Ce}^{*}$ 和 $(\mathrm{La} / \mathrm{Ce})_{\mathrm{N}}$ 的值 (网络版附录 3 ), 推测水井沱组时期构造环境为大陆边 缘. 整个水井沱组中 $\mathrm{Al}$ 与 $\mathrm{Th}$ 和 $\mathrm{Ti}$ 显著正相关 $\left(R_{\mathrm{Al}-\mathrm{Ti}}=0.94\right.$; $\left.R_{\mathrm{Al}-\mathrm{Th}}=0.95, P<0.01, n=16\right)$, 表明水井沱组中陆源碎屑 的物源区稳定.

水井沱组下部 $\mathrm{Se}$ 显著富集层中 $\mathrm{Se}$ 与 $\mathrm{Al}$ 显著正相 关 $\left(R_{\mathrm{Al}-\mathrm{Se}}=0.81, P<0.05, n=7\right)$, 表明该段层位中 Se含量主 要受陆源碎屑的控制. 该富集层中 $\mathrm{Se}$ 与 $\mathrm{TOC}$ 显示出较 强的负相关 $\left(R_{\mathrm{TOC}-\mathrm{Se}}=-0.65\right)$, 指示高有机质并未对 $\mathrm{Se}$ 的 富集有贡献. 该富集层中 $\mathrm{Fe}$ 与 $\mathrm{S}$ 具有显著正相关关系 $\left(R_{\mathrm{Fe}-\mathrm{S}}=0.81, P<0.01, n=7\right)$, 指示 $\mathrm{S}$ 主要为黄铁矿硫. 而 $\mathrm{Se}$ 与 $\mathrm{S}$ 表现出较强的负相关 $\left(R_{\mathrm{Se}-\mathrm{S}}=-0.49\right)$, 说明黄铁矿并 未对 $\mathrm{Se}$ 的富集有贡献. 该富集层中 $\mathrm{P}$ 与 $\mathrm{Al}$ 表现出非显 著的正相关关系 $\left(R_{\mathrm{Al}-\mathrm{P}}=0.43\right)$, 表明 $\mathrm{P}$ 受陆源输入的影响 不显著, 主要来源为深部物源. 而 $\mathrm{Se}$ 与 $\mathrm{P}$ 仅表现出一定 的非显著的正相关关系 $\left(R_{\mathrm{Se}-\mathrm{P}}=0.39\right)$, 指示 $\mathrm{Se}$ 可能还受 一定的深部物源的影响.

水井沱组上部 $\mathrm{Se}$ 显著富集层中 $\mathrm{Se}$ 与 $\mathrm{Al}$ 呈现较强的 正相关 $\left(R_{\mathrm{Al}-\mathrm{Se}}=0.60\right)$, 表明 $\mathrm{Se}$ 含量在一定程度上受到陆源 碎屑的影响, 同时说明还受别的因素的影响. 该富集层 中 Se与TOC具有显著正相关 $\left(R_{\mathrm{TOC}-\mathrm{Se}}=0.94, P<0.01, n=6\right)$, 表明高有机质对 $\mathrm{Se}$ 的显著富集贡献明显. 该富集层中 $\mathrm{Fe}$ 与 $\mathrm{S}$ 具有一定的正相关关系, 但不显著 $\left(R_{\mathrm{Fe}-\mathrm{S}}=0.66\right)$, $\mathrm{Se}$ 与 $\mathrm{S}$ 同样表现出非显著的正相关关系 $\left(R_{\mathrm{Fe}-\mathrm{S}}=0.36\right)$, 说 明黄铁矿的存在对 Se的富集有一定的贡献, 但不显著. 该富集层中 $\mathrm{P}$ 与 $\mathrm{Al}$ 具有微弱的负相关 $\left(R_{\mathrm{Al}-\mathrm{P}}=-0.03\right)$, 表 明 $\mathrm{P}$ 的主要来源为深部物源. 而 $\mathrm{Se}$ 与 $\mathrm{P}$ 具有较强的负相 关关系 $\left(R_{\mathrm{Se}-\mathrm{P}}=-0.70\right)$, 说明 $\mathrm{Se}$ 含量不受深部物源的影响.

整个水井沱组表现出轻微 $\mathrm{Eu}$ 正异常 $\left(\mathrm{Eu} / \mathrm{Eu}^{*}\right.$ 平均 $=1.12)$ (表 1). TOC 与 $\mathrm{Mo}$ 和 U 表现出显著正相关关系 ( $R_{\mathrm{TOC}-\mathrm{Mo}}=0.72, R_{\mathrm{TOC}-\mathrm{U}}=0.68, P<0.01, n=16$ ), 指示该组的 沉积环境为缺氧环境, 并未达到硫化的状态, 因此排 除硫化状态对 $\mathrm{Eu}$ 正异常的干扰. 轻微的Eu的正异常表
明水井沱组中受热液作用的影响并不显著. $\mathrm{K}_{2} \mathrm{O}-\mathrm{Rb}$ 图 解(图9a)指示, 水井沱组中仍受火山碎屑物质的影响, $\mathrm{Se}$ 可能具有火山碎屑的来源.

\subsection{7 其他硒的非显著富集层}

(1) 陡 I. 该段 $\mathrm{Se}$ 与 $\mathrm{Al}$ 和 $\mathrm{Th}$ 具有较弱的正相关关 系 $\left(R_{\mathrm{Se}-\mathrm{Al}}=0.21, R_{\mathrm{Se}-\mathrm{Th}}=0.16\right)$, 表明 $\mathrm{Se}$ 受陆源碎屑输入 影响小. $\mathrm{P}$ 与 $\mathrm{Al}$ 和 $\mathrm{Th}$ 具有较弱的正相关 $\left(R_{\mathrm{P}-\mathrm{Al}}=0.40\right.$, $\left.R_{\mathrm{P}-\mathrm{Th}}=0.41\right)$, 说明 $\mathrm{P}$ 受陆源影响较小, 可能受到深部物源 的影响, 这与当时的海侵一致(McFadden等, 2008; 田 兴磊等, 2014). $\mathrm{Se}$ 与 $\mathrm{P}$ 表现出微弱的负相关 $(R=-0.15)$, 表明 $\mathrm{Se}$ 受深部物源影响较小. 该段 $\mathrm{Eu}$ 异常以轻微正异 常为主 $\left(E u / \mathrm{Eu}^{*}\right.$ 平均 $\left.=1.41\right)$, 而在陡 $\mathrm{I}$ 底部 $0.4 \mathrm{~m}$ 处出现一次 明显的正异常 $\left(\mathrm{Eu} / \mathrm{Eu}^{*}=3.18\right)$, 研究认为该异常受到了 深海热液流的影响(田兴否等, 2014). 在该 $\mathrm{Eu}$ 明显正 异常的层位中, $\mathrm{Se}$ 的富集程度均出现峰值 $\left(\mathrm{Se}_{\mathrm{EF}}=1.00\right.$, $\left.\mathrm{Se}_{\mathrm{CC}}=0.71, \mathrm{Se}_{\mathrm{FF}}=15.99\right)$, 暗示 $\mathrm{Se}$ 具有热液来源. 而该层 位中 Se的富集程度低于陡II下部Se显著富集层中Se的 富集程度, 可能跟陡 $\mathrm{I}$ 盖帽白云岩沉积早期受到冰融 淡水稀释的影响(黄晶等, 2009; 田兴否等, 2014)有关. $\mathrm{K}_{2} \mathrm{O}-\mathrm{Rb}$ 图解(图9b)指示, 陡I中几乎无火山碎屑物质影 响, Se基本不受火山碎屑物质输入的影响.

(2) 陡II中部. 此段 Se非显著富集层中 Se含量受陆 源碎屑控制(讨论见4.3.1). Eu异常表现出轻微的正异 常, 未见明显的正异常, 表明热液作用不明显. 另外, 该段 $\mathrm{P}$ 的来源主要为深部海水的上涌. 陡 $\mathrm{II}$ 中部 $\mathrm{Se}$ 与 $\mathrm{P}$ 具有显著正相关关系 $(R=0.48, P<0.01)$, 表明 $\mathrm{Se}$ 的含量 可能受深部物源的控制. $\mathrm{K}_{2} \mathrm{O}-\mathrm{Rb}$ 图解(图9b)指示, 该 段部分层位中 $\mathrm{Se}$ 可能受火山碎屑物质输入的影响.

(3) 陡III下部及中部和上部. 前文分析表明(见 4.3.3) 陡III中 Se受陆源碎屑输入的作用显著, 并且 $\mathrm{Se}$ 不具有深部来源. 该段 $\mathrm{Se}$ 非显著富集层中 $\mathrm{Eu}$ 表现为轻 微的正异常或轻微的负异常, $\mathrm{Eu} / \mathrm{Eu}^{*}$ 的范围为 0.81 至 1.33 , 均值为 1.07 , 未见明显的正异常. 并且, 热液特征 元素Ba、 $\mathrm{Sr} 、 \mathrm{~Pb}$ 和 $\mathrm{Zn}$ 除个别层位外并未表现出明显 的增加, 说明热液作用并不明显. $\mathrm{K}_{2} \mathrm{O}-\mathrm{Rb}$ 图解(图9b) 指示, 该段未受明显的火山碎屑物质的影响.

(4) 灯影组. 该组中 $\mathrm{Se}$ 与 $\mathrm{Al}$ 和 Th表现出显著正相 关关系 $\left(R_{\mathrm{Se}-\mathrm{Al}}=0.69, R_{\mathrm{Se}-\mathrm{Th}}=0.53, P=0.01\right)$, 表明 $\mathrm{Se}$ 含量受 到陆源碎屑的影响显著. 该段中 $\mathrm{Eu}$ 表现为轻微的正异 常或轻微的负异常, $\mathrm{Eu} / \mathrm{Eu}^{*}$ 的范围为 $0.89 \sim 1.78$, 均值为 

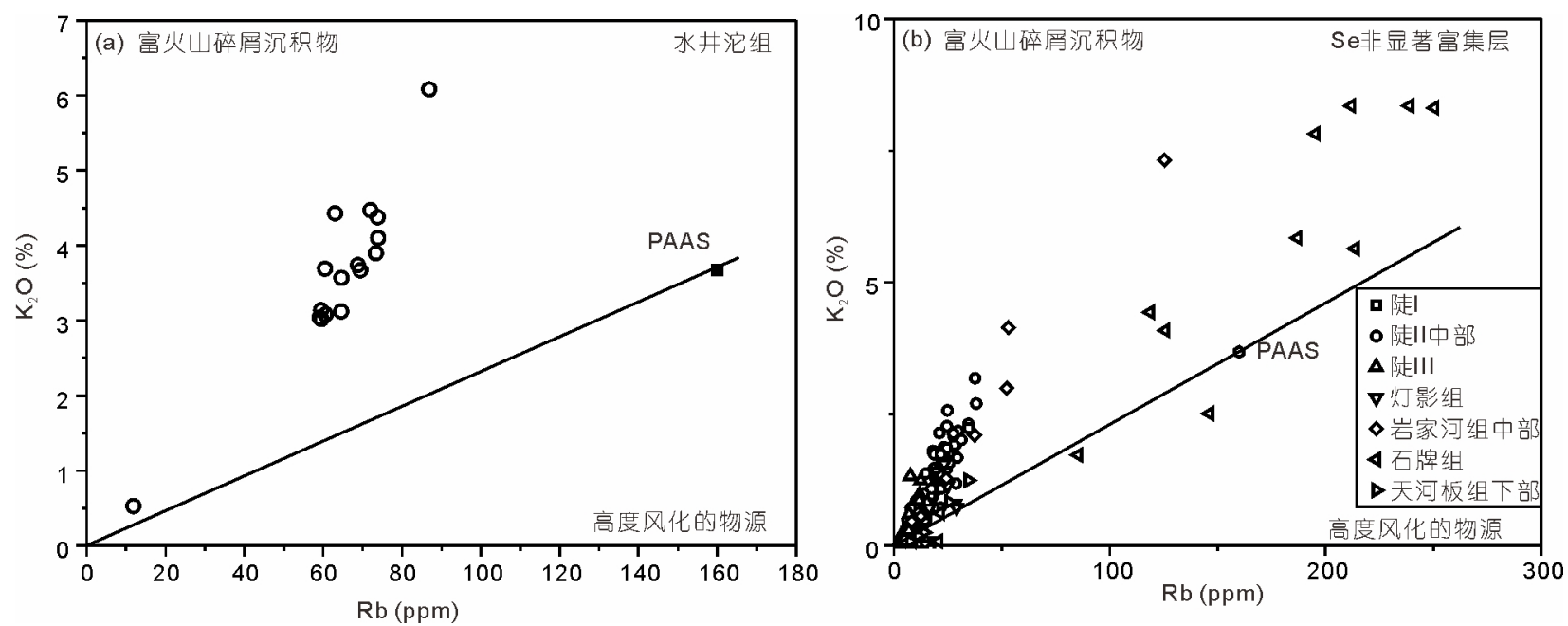

图 9 水井沱组(a)和 $\mathrm{Se}$ 非显著富集层(b)火山碎屑物质影响判别图解

1.06 , 无明显的正异常, 暗示热液作用存在但影响 不明显. 该组中 $\mathrm{P}$ 与 $\mathrm{Al}$ 和 $\mathrm{Th}$ 未表现出显著正相关关 系 $\left(R_{\mathrm{P}-\mathrm{Al}}=0.15, R_{\mathrm{P}-\mathrm{Th}}=0.03\right)$, 而 $\mathrm{Se}$ 与 $\mathrm{P}$ 无显著正相关关系 $(R=0.10)$, 指示 $\mathrm{Se}$ 不受深部物源影响. $\mathrm{K}_{2} \mathrm{O}-\mathrm{Rb}$ 图解(图 $9 b)$ 指示, 该组未受明显的火山碎屑物质的影响.

(5) 岩家河组中部. 岩家河组中Se受陆源物质的影 响不明显(见讨论4.3.4), 岩家河组中部 $\mathrm{Se}$ 与 $\mathrm{Al}$ 表现出 不显著的正相关性 $(R=0.67)$, 表明 $\mathrm{Se}$ 受陆源碎屑影响, 但不明显. 岩家河组中部 $\mathrm{Eu}$ 表现为轻微正异常或基本 无异常 $\left(\mathrm{Eu} / \mathrm{Eu}^{*}\right.$ 均值 $\left.=1.11\right)$ 以及硅质岩 $(\mathrm{YJH}-21)$ 的非热水 成因(图7), 表明热液作用并不十分明显. $\mathrm{K}_{2} \mathrm{O}-\mathrm{Rb}$ 图解 (图9b)指示, 该段可能仍受部分火山碎屑物质的影响. 该段中 $\mathrm{P}$ 与 $\mathrm{Al}$ 和 $\mathrm{Th}$ 显著正相关 $\left(R_{\mathrm{P}-\mathrm{Al}}=0.88, R_{\mathrm{P}-\mathrm{Th}}=0.92\right.$, $P<0.01)$, 推测 $\mathrm{P}$ 的不受深部物源控制, 而 $\mathrm{Se}$ 与 $\mathrm{P}$ 表现出 显著的正相关性 $(R=0.83, P<0.05)$, 表明 $\mathrm{Se}$ 不具有深部 物源.

(6) 水井沱组中部. 该段中 $\mathrm{Se}$ 与 $\mathrm{Al}$ 表现出负相关性 $(R=-0.22)$, 表明 $\mathrm{Se}$ 含量不受陆源碎屑的影响.水井沱组 中部 $\mathrm{Eu}$ 表现为轻微正异常或无异常 $\left(\mathrm{Eu} / \mathrm{Eu}^{*}{ }^{*}\right.$ 均值 $\left.=1.06\right)$, 指示热液作用并不十分明显. $\mathrm{K}_{2} \mathrm{O}-\mathrm{Rb}$ 图解(图9a)指示, 该段仍受部分火山碎屑物质的影响. 该段中 $\mathrm{P}$ 与 $\mathrm{Al}$ 表 现出较强的负相关性 $\left(R_{\mathrm{P}-\mathrm{Al}}=-0.82\right)$, 推测 $\mathrm{P}$ 的含量受深 部物源控制, 而 $\mathrm{Se}$ 与 $\mathrm{P}$ 具有一定的负相关性 $(R=-0.37)$, 因此 Se不具有深部物源.

(7) 石牌组. 该组中 $\mathrm{Se}$ 与 $\mathrm{Al}$ 和 $\mathrm{Th}$ 表现出微弱的负 相关关系 $\left(R_{\mathrm{Se}-\mathrm{Al}}=-0.11, R_{\mathrm{Se}-\mathrm{Th}}=-0.19\right)$, 表明 $\mathrm{Se}$ 基本不受
陆源碎屑输入的影响. $\mathrm{P}$ 与 $\mathrm{Al}$ 表现出较弱的正相关 $\left(R_{\mathrm{P}-\mathrm{Al}}=0.21\right)$, 指示 $\mathrm{P}$ 受到陆源碎屑输入的影响, 主要受 深部物源的控制. 而 $\mathrm{Se}$ 与 $\mathrm{P}$ 基本无相关性 $(R=-0.01)$, 推 测 Se受深部物源的控制不明显. 石牌组下部 $\mathrm{Eu}$ 具有轻 微的正异常 $\left(\mathrm{Eu} / \mathrm{Eu}^{*}\right.$ 平均=1.05), 而石牌组上部 $\mathrm{Eu}$ 表现为 微弱的负异常 $\left(\mathrm{Eu} / \mathrm{Eu}^{*}\right.$ 平均 $\left.=0.96\right)$, 暗示该组中热液作用 的影响开始变弱, 甚至基本无热液作用的影响. $\mathrm{K}_{2} \mathrm{O}-\mathrm{Rb}$ 图解(图9b)指示石牌组样品 $\mathrm{K}_{2} \mathrm{O}-\mathrm{Rb}$ 落在PAAS附近, 表 明基本不受火山碎屑物质的影响, 因此 $\mathrm{Se}$ 基本无火山 碎屑的物源.

(8) 天河板组下部. 该段中 $\mathrm{Se}$ 与 $\mathrm{Al}$ 和 $\mathrm{Th}$ 表现出微弱 的负相关关系 $\left(R_{\mathrm{Se}-\mathrm{Al}}=-0.34, R_{\mathrm{Se}-\mathrm{Th}}=-0.04\right)$, 表明 $\mathrm{Se}$ 基本 不受陆源碎屑输入的影响. 该段 $\mathrm{K}_{2} \mathrm{O}-\mathrm{Rb}$ 落在PAAS附 近(图9b), 表明基本不受火山碎屑物质的影响. $\mathrm{P}$ 与 $\mathrm{Al}$ 表现出较弱的正相关 $\left(R_{\mathrm{P}-\mathrm{Al}}=0.31\right)$, 但均不显著, 指示 $\mathrm{P}$ 含量受到陆源碎屑的影响, 主要受深部物源的控制. 而 $\mathrm{Se}$ 与 $\mathrm{P}$ 基本无相关性 $(R=0.09)$, 暗示 $\mathrm{Se}$ 受深部物源影响 较小. 该段中 $\mathrm{Eu}$ 表现为微弱的负异常 $\left(\mathrm{Eu} / \mathrm{Eu}^{*}\right.$ 平均 $\left.=0.87\right)$, 表明无热液活动的影响.

\section{4 氧化还原条件对硒富集的可能影响}

在用微量元素判别沉积环境的氧化还原条件之前, 首先必须评估碎屑物质的影响(Tribovillard等, 2006). 根据前文4.3.1中所述, 将主要关注的氧化还原指标元 素 $\mathrm{V} 、 \mathrm{Cr} 、 \mathrm{Ni}$ 和U与 $\mathrm{Th} 、 \mathrm{Al}$ 做相关性(表4). 对其中显 示出显著正相关关系的元素 $X$ 的自生含量按公式 $X_{\text {碎首 }}=$ 
表 4 三峡地区埃迪卡拉系至下寒武统不同组段中氧化还原敏感元素与陆源指示元素的相关性分析

\begin{tabular}{|c|c|c|c|c|c|c|c|c|c|c|c|c|}
\hline \multirow[b]{2}{*}{ 元素 } & \multicolumn{2}{|c|}{ 陡 I } & \multicolumn{2}{|c|}{ 陡 II } & \multicolumn{2}{|c|}{ 陡 III } & \multicolumn{2}{|c|}{ 陡IV } & \multicolumn{2}{|c|}{ 蛤蟆井段 } & \multicolumn{2}{|c|}{ 石板滩段 } \\
\hline & $\mathrm{Al}$ & Th & $\mathrm{Al}$ & Th & $\mathrm{Al}$ & Th & $\mathrm{Al}$ & Th & $\mathrm{Al}$ & Th & $\mathrm{Al}$ & Th \\
\hline V & $0.96^{* *}$ & $0.64^{*}$ & $0.95^{* *}$ & $0.79^{* *}$ & $0.53^{* *}$ & $0.62^{* *}$ & 0.53 & 0.25 & $0.90^{*}$ & 0.77 & -0.17 & -0.07 \\
\hline $\mathrm{Cr}$ & $0.73^{* *}$ & $0.75^{*}$ & $0.53^{* *}$ & $0.44^{* *}$ & -0.16 & -0.08 & $0.63^{*}$ & -0.08 & 0.90 & 0.72 & $0.51^{*}$ & $0.51^{*}$ \\
\hline $\mathrm{Ni}$ & -0.51 & -0.06 & $0.46^{* *}$ & $0.38^{* *}$ & -0.33 & -0.24 & -0.08 & 0.44 & $0.87^{*}$ & $0.90^{*}$ & $0.71^{* *}$ & $0.70^{* *}$ \\
\hline \multirow[t]{2}{*}{$\mathrm{U}$} & $-0.75^{*}$ & -0.30 & $0.38^{* *}$ & $0.72^{* *}$ & $0.40^{*}$ & $0.45^{* *}$ & 0.11 & -0.06 & 0.63 & 0.44 & 0.10 & 0.08 \\
\hline & \multicolumn{2}{|c|}{ 白马沱段 } & \multicolumn{2}{|c|}{ 岩家河组 } & \multicolumn{2}{|c|}{ 水井沱组 } & \multicolumn{2}{|c|}{ 石牌组 } & \multicolumn{2}{|c|}{ 天河板组下部 } & & \\
\hline 元素 & $\mathrm{Al}$ & Th & $\mathrm{Al}$ & Th & $\mathrm{Al}$ & Th & $\mathrm{Al}$ & Th & $\mathrm{Al}$ & Th & & \\
\hline V & 0.07 & 0.15 & $0.51^{* *}$ & $0.46^{*}$ & 0.32 & 0.34 & $0.97^{* *}$ & $0.82^{* *}$ & $0.92^{*}$ & 0.70 & & \\
\hline $\mathrm{Cr}$ & $0.98^{* *}$ & $0.98^{* *}$ & $0.59^{* *}$ & $0.52^{* *}$ & $0.71^{* *}$ & $0.70^{* *}$ & $0.94^{* *}$ & $0.83^{* *}$ & $0.91^{*}$ & 0.87 & & \\
\hline $\mathrm{Ni}$ & $0.52^{*}$ & $0.56^{* *}$ & $0.62^{* *}$ & $0.53^{* *}$ & 0.13 & 0.10 & $0.99^{* *}$ & $0.83^{* *}$ & $0.91^{*}$ & $0.94^{*}$ & & \\
\hline U & -0.06 & -0.04 & $0.49^{* *}$ & $0.52^{* *}$ & 0.22 & 0.11 & $0.64^{*}$ & $0.82^{* *}$ & 0.08 & 0.45 & & \\
\hline
\end{tabular}

$* P<0.05 ; * * P<0.01$

$(X / \mathrm{Al})_{\text {上地壳 }} \times \mathrm{Al}_{\text {样品 }}, X_{\text {自生 }}=X_{\text {总 }} X_{\text {碎屏 }}$ (Tribovillard等, 2006), 其中上地壳的含量引自(McLennan, 2001), 进 行处理之后, 运用微量元素自生值的相关比值重建古 海洋沉积水体氧化还原环境.

$\mathrm{Th}$ 和 $\mathrm{U}$ 在还原状态下地球化学性质相似, 在氧 化状态下差别很大(Wedepohl和Correns, 1969; Guo等, 2007; Pi等, 2014). 在表生环境下, Th只有 +4 价一种价 态且不易溶解, 而U则不一样. U在强还原状态下为 +4 价, 不溶解于水, 导致它在沉积物中富集; 而在氧 化状态下, U以易溶的 +6 价存在, 造成沉积物中U的丢 失. 依据U、Th化学行为差异, $U / T h$ 可以作为鉴别氧 化还原环境的一个参数, 在缺氧(还原)环境下, $\mathrm{U} / \mathrm{Th}$ 大 于 1.25 , 而在贫氧条件下在 $0.75 \sim 1.25$, 氧化条件下小于 0.75(Jones和Manning, 1994; Rimmer等, 2004; Pattan等, 2005). U/Th作为一类重要的古环境指标被广泛应用于 沉积环境的氧化还原条件的判定中(Jones和Manning, 1994; Rimmer等, 2004; Pattan等, 2005).

钒是一种优先在缺氧和近似缺氧水体中富集的 氧化还原敏感元素(Calvert和Pedersen, 1993). $\mathrm{V} / \mathrm{Cr}$ 和 $\mathrm{V} /(\mathrm{V}+\mathrm{Ni})$ 一直被用作为判别古环境氧化还原条件的重 要指标(Hatch和Leventhal, 1992; Jones和Manning, 1994; Algeo和Maynard, 2004; Rimmer, 2004; Rimmer等, 2004; Guo等, 2007; Pi等, 2014). Jones和Manning(1994)把 V/Cr 小于 2 指示氧化环境, 2 至 4.25 指示贫氧环境, 大于 4.25 指示次氧化的至缺氧环境. $\mathrm{V} /(\mathrm{V}+\mathrm{Ni})$ 不仅能反映沉积
物的沉积环境, 还能反映古海洋水体的分层性, 其中 $\mathrm{V} /(\mathrm{V}+\mathrm{Ni})$ 大于 0.84 指示为静海相还原环境, 而且古海 洋水体分层强烈, 小于 0.6 指示古海洋水体呈弱分层的 贫氧环境(Hatch和Leventhal, 1992; Rimmer, 2004).

在陡II底部 Se显著富集层, U/Th和 $\mathrm{V} / \mathrm{Cr}$ 指示沉积 环境为氧化的至贫氧的环境(表 2 ; 图 2$), \mathrm{V} /(\mathrm{V}+\mathrm{Ni})$ 指示 海洋水体分层较强, 而部分显示强烈的分层. 陡II上部 $\mathrm{Se}$ 显著富集层, 整体上 $\mathrm{U} / \mathrm{Th}$ 和 $\mathrm{V} / \mathrm{Cr}$ 指示沉积环境为氧 化的至贫氧的环境, $\mathrm{V} /(\mathrm{V}+\mathrm{Ni})$ 指示水体分层性较弱(表 2 ; 图2). 陡III顶部 $\mathrm{Se}$ 显著富集层, $\mathrm{V} / \mathrm{Cr}$ 和 $\mathrm{V} /(\mathrm{V}+\mathrm{Ni})$ 指示 沉积环境为缺氧的并且分层性强的沉积环境, $U / T h$ 指 示贫氧的沉积环境. 陡IV的Se显著富集层, 3 个指标整 体上指示缺氧的分层性较强的环境, 同时指示发生过 贫氧的波动事件(表2; 图2).

对于岩家河底部Se显著富集层, $\mathrm{U} / \mathrm{Th}$ 指示缺氧环 境, $\mathrm{V} / \mathrm{Cr}$ 指示氧化环境, $\mathrm{V} /(\mathrm{V}+\mathrm{Ni}$ )指示弱分层(表2; 图 $2)$. 岩家河组上部 $\mathrm{Se}$ 显著富集层 $U / T h$ 和 $V / C r$ 指示沉积 环境氧化至贫氧的波动转变, 在岩家河组顶部及随后 的水井沱组下部又指示为分层较强的缺氧环境, 水井 沱组中部沉积环境以较强分层的氧化条件为主, 之后 在水井沱组上部 $(\mathrm{Se}$ 富集层)为缺氧环境(表 2 , 图 2$)$.

尽管用微量元素比值判断氧化还原条件在个别 层位中有一定的矛盾, 但仍可以看出, $\mathrm{Se}$ 的显著富集 与贫氧及缺氧环境有一定的关系, 在缺氧条件下更利 于富集. 但仅仅用微量元素比值判断古环境的氧化还 
原条件出现一定的矛盾, 今后还需用其他的指标等进 一步研究古环境的氧化还原条件, 进而讨论氧化还原 条件与 $\mathrm{Se}$ 的富集的关系.

\section{5 结论}

(1) 相对于上地壳微量元素均值, Se 是三峡地区 埃迪卡拉系至下寒武统 $\left(E-C_{1}\right)$ 中所研究的 23 种微量元 素中最富集的元素, 其富集系数 $\left(\mathrm{Se}_{\mathrm{EF}}=26.97\right)$ 显著强于 其他微量元素, 是第二富集元素 $\mathrm{Cd}\left(\mathrm{Cd}_{\mathrm{EF}}=10.66\right)$ 的 2.53 倍, 并且Se在埃迪卡拉纪至早寒武世地层中富集系数 $\mathrm{EF}$ 的变化较大, 其富集系数 $\mathrm{EF}$ 的差值为601.2. 浓集系 数 (与同类岩性相比)均值表明, $\mathrm{Se}$ 在三峡地区 $\mathrm{E}-\mathrm{C}_{1}$ 地 层中具有最高的 $\mathrm{CC}$ 值 $\left(\mathrm{Se}_{\mathrm{CC}}=48.04\right)$, 是第二浓集微量 元素 $\mathrm{Cd}\left(\mathrm{Cd}_{\mathrm{CC}}=10.70\right)$ 的 4.49 倍. 富集系数 $\mathrm{EF}^{\prime}(\mathrm{Al}$ 标准化 之后的富集系数)指示, $\mathrm{Se}$ 为第二富集的元素, $\mathrm{EF}^{\prime}$ 均值 为 218.73 , 稍微小于 $\mathrm{Cd}\left(\mathrm{Cd}_{\mathrm{EF}}=288.46\right)$, 但远高于第三富 集的微量元素As( $\left.\mathrm{As}_{\mathrm{EF}}=97.49\right)$.

(2) $\mathrm{Se}$ 在该地区 $\mathrm{E}-\mathrm{C}_{1}$ 地层中含量变化范围 为 $<10^{-5} \sim 30.08 \mathrm{ppm}$, 算数均值为 $1.35 \mathrm{ppm}$. 其平 均富集系数 $\mathrm{EF}$ 呈现为水井沱组 $\left(\mathrm{Se}_{\mathrm{EF}}=92.58\right)>$ 岩 家河组 $\left(\mathrm{Se}_{\mathrm{EF}}=54.45\right)>$ 陡山沱组 $\left(\mathrm{Se}_{\mathrm{EF}}=24.72\right)>$ 灯影 组 $\left(\mathrm{Se}_{\mathrm{EF}}=2.48\right)>$ 石牌组 $\left(\mathrm{Se}_{\mathrm{EF}}=1.95\right)>$ 天河板组下部 $\left(\mathrm{Se}_{\mathrm{EF}}=1.24\right)$. 其中在水井沱组、岩家河组和陡山沱组 中的富集系数 $\mathrm{EF}$ 均值远高于其他组. 在整个涉及的 E- $\mathrm{C}_{1}$ 的地层中, $\mathrm{Se}$ 的平均富集系数 $\mathrm{EF}$ 值为 26.97 , 是下 伏成冰系南沱组 $\mathrm{Se}$ 平均富集系数 $\mathrm{EF}$ 的 11.78 倍.

(3) Q-Q图和频率分布直方图指示三峡地区E- $C_{1}$ 地 层中 Se的含量服从自然对数正态分布, 并且表现出正偏 态分布类型, 即低浓度样品数量居于多数 $(<2.5 \mathrm{ppm})$, 而 高浓度主要出现在个别层位内. 而Se显著富集(EF>10) 的层位主要包括陡II下部、陡II上部、陡III顶部、陡 IV、岩家河组底部和上部、水井沱组下部和上部.

(4) Se显著富集层的元素地球化学特征表明: 陡II 下部Se含量受陆源碎屑、热液及火山碎屑的影响, 并 且有机质对 Se的富集有贡献. 陡II上部Se含量受陆源 碎屑的影响和火山碎屑的影响, 此外, 黄铁矿促进 $\mathrm{Se}$ 的 富集. 陡III顶部 $\mathrm{Se}$ 含量受陆源碎屑和热液的影响. 陡 IV中Se含量在一定程度上受陆源碎屑的影响, 但不显 著, 此外 Se还受火山碎屑源的影响, 并且黄铁矿对 $\mathrm{Se}$ 的富集有一定的贡献. 岩家河组底部 $\mathrm{Se}$ 受陆源碎屑、 火山碎屑和深部物源的影响, 而岩家河组上部 $\mathrm{Se}$ 含量
受到陆源碎屑、热液和火山碎屑的影响. 水井沱组下 部Se含量主要受陆源碎屑的影响, 此外还受部分深部 物源和火山碎屑的影响, 而水井沱组上部高有机质对 Se的富集有促进作用, 其含量受陆源碎屑和火山碎屑 的影响, 此外, 黄铁矿对 Se的富集有一定的贡献. Se的 非显著富集层的元素地球化学特征表明: 陡I中 Se含 量受热液影响. 陡II中部 $\mathrm{Se}$ 含量受陆源碎屑显著, 此 外还受火山碎屑的影响. 陡III下部、中部和上部 $\mathrm{Se}$ 含 量受陆源碎屑影响显著. 灯影组 $\mathrm{Se}$ 含量受陆源碎屑影 响显著. 岩家河组中部Se受陆源碎屑和火山物质的影 响. 水井沱组中部 $\mathrm{Se}$ 含量受火山碎屑影响, 并且有机 质对 $\mathrm{Se}$ 的富集有一定的促进作用. 石牌组和天河板组 下部中 Se基本不受外源因素的影响.

(5) 通过与氧化还原敏感元素的比值 $\mathrm{U} / \mathrm{Th} 、 \mathrm{~V} / \mathrm{Cr}$ 和 $\mathrm{V} /(\mathrm{V}+\mathrm{Ni})$ 等反映的氧化还原条件的对比, 发现该时 期该地区 Se的显著富集与贫氧环境有一定的关系, 在 缺氧条件下更利于富集.

致谢中国科学院地理科学与资源研究所郭庆军研 究员在岩石总有机碳测试中给予指导和帮助, 中国科学 院地理科学与资源研究所理化分析中心张宏志高工在 ICP-OES和ICP-MS 实验测试过程中给予指导, 王少涁博 士和倪润祥博士在采样过程中提供帮助, 在此一并致谢.

\section{参考文献}

迟清华, 鄢明才. 2007. 应用地球化学元素丰度数据手册. 北京: 地 质出版社

丁振举, 刘丛强, 姚书振, 周宗桂. 2000. 海底热液系统高温流体的 稀土元素组成及其控制因素. 地球科学进展, 15: 68-73

黄晶, 储雪蕾, 常华进, 冯连君. 2009. 三峡地区埃迪卡拉系陡山 沱组帽碳酸盐岩的微量元素和稀土元素研究. 科学通报, 54 : 3498-3506

柳永清, 旷红伟, 彭楠. 2009. 峡东伊迪卡拉纪陡山沱组顶部重晶石 特征与地质意义研究. 地球学报, 30: 487-494

田兴磊, 雒昆利, 王少涁, 倪润祥. 2014. 长江三峡地区成冰纪-埃迪 卡拉纪转换时期微量元素和稀土元素地球化学特征. 古地理学 报, 16: 483-502

项礼文, 朱兆玲, 李善姬. 1999. 中国地层典 寒武系. 北京: 地质出 版社

问斌, 朱祥坤, 张飞飞, 唐索寒. 2014. 峡东地区埃迪卡拉系黑色页 岩的微量元素和Fe同位素特征及其古环境意义. 地质学报, 88 : 1603-1615

尹崇玉, 刘鹏举, 陈寿铭, 唐烽, 高林志, 王自强. 2009. 峡东地区埃迪 
卡拉系陡山沱组疑源类生物地层序列. 古生物学报, 48: 146-154 赵自强, 邢裕盛, 马国干. 1985. 长江三峡地区生物地层学(1). 震旦 纪分册. 北京: 地质出版社

Adachi M, Yamamoto K, Sugisaki R. 1986. Hydrothermal chert and associated siliceous rocks from the northern Pacific their geological significance as indication od ocean ridge activity. Sediment Geol, 47: $125-148$

Algeo T J, Maynard J B. 2004. Trace-element behavior and redox facies in core shales of Upper Pennsylvanian Kansas-type cyclothems. Chem Geol, 206: 289-318

Alibo D S, Nozaki Y. 1999. Rare earth elements in seawater: Particle association, shale-normalization, and Ce oxidation. Geochim Cosmochim Acta, 63: 363-372

Auclair G, Fouquet Y, Bohn M. 1987. Distribution of selenium in hightemperature hydrothermal sulfide deposits at $13^{\circ} \mathrm{N}$, East Pacific Rise. Can Mineral, 25: 577-587

Bau M, Dulski P. 1996. Distribution of yttrium and rare-earth elements in the Penge and Kuruman iron-formations, Transvaal Supergroup, South Africa. Precambrian Res, 79: 37-55

Boström K. 1983. Genesis of Ferromanganese Deposits-Diagnostic Criteria for Recent and Old Deposits. New York: Plenum Press. 473-489

Brookins D. 1989. Aqueous geochemistry of rare earth elements. Rev Mineral Geochem, 21: 201-225

Calvert S E, Pedersen T F. 1993. Geochemistry of Recent oxic and anoxic marine sediments: Implications for the geological record. Mar Geol, 113: $67-88$

Chen Z, Zhou C M, Meyer M, Xiang K, Schiffbauer J D, Yuan X L, Xiao S H. 2013. Trace fossil evidence for Ediacaran bilaterian animals with complex behaviors. Precambrian Res, 224: 690-701

Condon D, Zhu M Y, Bowring S, Wang W, Yang A H, Jin Y G. 2005. $\mathrm{U}-\mathrm{Pb}$ ages from the Neoproterozoic Doushantuo Formation, China. Science, 308: 95-98

Filippelli G M. 2008. The global phosphorus cycle: Past, present, and future. Elements, 4: 89-95

Floor G H, Román-Ross G. 2012. Selenium in volcanic environments: A review. Appl Geochem, 27: 517-531

Guo J F, Li Y, Li G X. 2014. Small shelly fossils from the early Cambrian Yanjiahe Formation, Yichang, Hubei, China. Gondwana Res, 25: 999-1007

Guo Q J, Shields G A, Liu C Q, Strauss H, Zhu M Y, Pi D H, Goldberg T, Yang X L. 2007. Trace element chemostratigraphy of two Ediacaran-Cambrian successions in South China: Implications for organosedimentary metal enrichment and silicification in the Early Cambrian. Palaeogeogr Palaeoclimatol Palaeoecol, 254: 194-216

Hatch J R, Leventhal J S. 1992. Relationship between inferred redox po- tential of the depositional environment and geochemistry of the Upper Pennsylvanian (Missourian) Stark Shale Member of the Dennis Limestone, Wabaunsee County, Kansas, U.S.A. Chem Geol, 99: 65-82

Hoffman P F, Kaufman A J, Halverson G P, Schrag D P. 1998. A Neoproterozoic snowball Earth. Science, 281: 1342-1346

Ishikawa T, Ueno Y, Komiya T, Sawaki Y, Han J, Shu D G, Li Y, Maruyama S, Yoshida N. 2008. Carbon isotope chemostratigraphy of a Precambrian/Cambrian boundary section in the Three Gorge area, South China: Prominent global-scale isotope excursions just before the Cambrian Explosion. Gondwana Res, 14: 193-208

Ishikawa T, Ueno Y, Shu D, Li Y, Han J, Guo J, Yoshida N, Komiya T. 2013. Irreversible change of the oceanic carbon cycle in the earliest Cambrian: High-resolution organic and inorganic carbon chemostratigraphy in the Three Gorges area, South China. Precambrian Res, 225: 190-208

Jiang G Q, Kaufman A J, Christie-Blick N, Zhang S H, Wu H C. 2007. Carbon isotope variability across the Ediacaran Yangtze platform in South China: Implications for a large surface-to-deep ocean $\delta^{13} \mathrm{C}$ gradient. Earth Planet Sci Lett, 261: 303-320

Jiang G Q, Shi X Y, Zhang S H, Wang Y, Xiao S H. 2011. Stratigraphy and paleogeography of the Ediacaran Doushantuo Formation (ca. 635-551 Ma) in South China. Gondwana Res, 19: 831-849

Jones B, Manning D A C. 1994. Comparison of geochemical indices used for the interpretation of palaeoredox conditions in ancient mudstones. Chem Geol, 111: 111-129

Large R R, Halpin J A, Danyushevsky L V, Maslennikov V V, Bull S W, Long J A, Gregory D D, Lounejeva E, Lyons T W, Sack P J, McGoldrick P J, Calver C R. 2014. Trace element content of sedimentary pyrite as a new proxy for deep-time ocean-atmosphere evolution. Earth Planet Sci Lett, 389: 209-220

Ling H F, Chen X, Li D, Wang D, Shields-Zhou G A, Zhu M Y. 2013. Cerium anomaly variations in Ediacaran-earliest Cambrian carbonates from the Yangtze Gorges area, South China: Implications for oxygenation of coeval shallow seawater. Precambrian Res, 225: $110-127$

Liu P J, Yin C Y, Chen S M, Tang F, Gao L Z. 2013. The biostratigraphic succession of acanthomorphic acritarchs of the Ediacaran Doushantuo Formation in the Yangtze Gorges area, South China and its biostratigraphic correlation with Australia. Precambrian Res, 225: 29-43

Liu P J, Yin C Y, Gao L Z, Tang F, Chen S M. 2009. New material of microfossils from the Ediacaran Doushantuo Formation in the Zhangcunping area, Yichang, Hubei Province and its zircon SHRIMP U-Pb age. Chin Sci Bull, 54: 1058-1064

Luo K L. 2011. Arsenic and fluorine contents and distribution patterns of Early Paleozoic stonelike coal in the Daba Fold Zone and Yangtze Plate, China. Energy Fuels, 25: 4479-4487 
Luo K L, Xu L R, Tan J A, Wang D H, Xiang L H. 2004. Selenium source in the selenosis area of the Daba region, South Qinling Mountain, China. Environ Geol, 45: 426-432

Marchig V, Gundlach H, Möller P, Schley F. 1982. Some geochemical indicators for discrimination between diagenetic and hydrothermal metalliferous sediments. Mar Geol, 50: 241-256

McFadden K A, Huang J, Chu X, Jiang G, Kaufman A J, Zhou C, Yuan X, Xiao S. 2008. Pulsed oxidation and biological evolution in the Ediacaran Doushantuo Formation. Proc Natl Acad Sci USA, 105: 3197-3202

McFadden K A, Xiao S H, Zhou C M, Kowalewski M. 2009. Quantitative evaluation of the biostratigraphic distribution of acanthomorphic acritarchs in the Ediacaran Doushantuo Formation in the Yangtze Gorges area, South China. Precambrian Res, 173: 170-190

McLennan S M. 2001. Relationships between the trace element composition of sedimentary rocks and upper continental crust. Geochem Geophys Geosyst, 2: 1021-24

Michard A, Albarède F. 1986. The REE content of some hydrothermal fluids. Chem Geol, 55: 51-60

Murray R W. 1994. Chemical criteria to identify the depositional environment of chert: General principles and applications. Sediment Geol, 90: 213-232

Murray R W, Buchholtz ten Brink M R, Jones D L, Gerlach D C, Russ III G P. 1990. Rare earth elements as indicators of different marine depositional environments in chert and shale. Geology, 18: 268-271

Nance W B, Taylor S R. 1976. Rare earth element patterns and crustal evolution-I. Australian post-Archean sedimentary rocks. Geochim Cosmochim Acta, 40: 1539-1551

Ni R X, Luo K L, Tian X L, Yan S G, Zhong J T, Liu M Q. 2016. Distribution and geological sources of selenium in environmental materials in Taoyuan County, Hunan Province, China. Environ Geochem Health, 38: $927-938$

Niu C X, Luo K L. 2011. Relationship of selenium, arsenic and sulfur in soil and plants in Enshi County. China. J Food Agric Environ, 9: 646-651

Nozaki Y. 1997. A fresh look at element distribution in the North Pacific Ocean. Eos Trans AGU, 78: 221

Okada Y, Sawaki Y, Komiya T, Hirata T, Takahata N, Sano Y, Han J, Maruyama S. 2014. New chronological constraints for Cryogenian to Cambrian rocks in the Three Gorges, Weng'an and Chengjiang areas, South China. Gondwana Res, 25: 1027-1044

Olivarez A M, Owen R M. 1991. The europium anomaly of seawater: Implications for fluvial versus hydrothermal REE inputs to the oceans. Chem Geol, 92: 317-328

Pattan J N, Pearce N J G, Mislankar P G. 2005. Constraints in using Cerium-anomaly of bulk sediments as an indicator of paleo bottom water redox environment: A case study from the Central Indian Ocean Basin. Chem Geol, 221: 260-278

Pi D H, Jiang S Y, Luo L, Yang J H, Ling H F. 2014. Depositional environments for stratiform witherite deposits in the Lower Cambrian black shale sequence of the Yangtze Platform, southern Qinling region, SW China: Evidence from redox-sensitive trace element geochemistry. Palaeogeogr Palaeoclimatol Palaeoecol, 398: 125-131

Pi D H, Liu C Q, Shields-Zhou G A, Jiang S Y. 2013. Trace and rare earth element geochemistry of black shale and kerogen in the early Cambrian Niutitang Formation in Guizhou Province, South China: Constraints for redox environments and origin of metal enrichments. Precambrian Res, 225: 218-229

Plank T, Langmuir C H. 1998. The chemical composition of subducting sediment and its consequences for the crust and mantle. Chem Geol, 145: $325-394$

Plant J A, Bone J, Voulvoulis N, Kinniburgh D G, Smedley P L, Fordyce F M, Klinck B. 2014. 11.2 arsenic and selenium. In: Turekian H D H K, ed. Treatise on Geochemistry. 2nd ed. Oxford: Elsevier. 13-57

Rawlins B, McGrath S, Scheib A, Breward N, Cave M, Lister T, Ingham M, Gowing C, Carter S. 2012. The Advanced Soil Geochemical Atlas of England and Wales. British Geological Survey

Rimmer S M. 2004. Geochemical paleoredox indicators in DevonianMississippian black shales, Central Appalachian Basin (USA). Chem Geol, 206: 373-391

Rimmer S, Thompson J, Goodnight S, Robl T. 2004. Multiple controls on the preservation of organic matter in Devonian-Mississippian marine black shales: Geochemical and petrographic evidence. Palaeogeogr Palaeoclimatol Palaeoecol, 215: 125-154

Rotruck J T, Pope A L, Ganther H E, Swanson A B, Hafeman D G, Hoekstra W G. 1973. Selenium: Biochemical role as a component of glutathione peroxidase. Science, 179: 588-590

Scott C, Lyons T W, Bekker A, Shen Y, Poulton S W, Chu X, Anbar A D. 2008. Tracing the stepwise oxygenation of the Proterozoic ocean. Nature, 452: 456-459

Sverjensky D A. 1984. Europium redox equilibria in aqueous solution. Earth Planet Sci Lett, 67: 70-78

Taylor S R, McLennan S M. 1985. The Continental Crust: Its Composition and Evolution. Oxfold: Blackwell. 312

Tian X L, Luo K L. 2017. Selenium, arsenic and molybdenum variation and bio-radiation in the Ediacaran-Cambrian interval. Precambrian Res, 292: 378-385

Tribovillard N, Algeo T J, Lyons T, Riboulleau A. 2006. Trace metals as paleoredox and paleoproductivity proxies: An update. Chem Geol, 232: $12-32$

van Kranendonk M J, Webb G E, Kamber B S. 2003. Geological and trace element evidence for a marine sedimentary environment of de- 
position and biogenicity of $3.45 \mathrm{Ga}$ stromatolitic carbonates in the Pilbara Craton, and support for a reducing Archaean ocean. Geobiology, 1: 91-108

Wedepohl K H, Correns C W. 1969. Handbook of Geochemistry. Berlin: Springer Verlag

Yin C Y, Tang F, Liu Y Q, Gao L Z, Liu P J, Xing Y S, Yang Z Q, Wan Y S, Wang Z Q. 2005. U-Pb zircon age from the base of the Ediacaran Doushantuo Formation in the Yangtze Gorges, South China:
Constraint on the age of Marinoan glaciation. Episodes, 28: 48-49 Zhang C, Manheim F T, Hinde J, Grossman J N. 2005. Statistical characterization of a large geochemical database and effect of sample size. Appl Geochem, 20: 1857-1874

Zhu M Y, Lu M, Zhang J M, Zhao F C, Li G X, Yang A H, Zhao X, Zhao M J. 2013. Carbon isotope chemostratigraphy and sedimentary facies evolution of the Ediacaran Doushantuo Formation in western Hubei, south China. Precambrian Res, 225: 7-28 